\title{
Coronavirus-specific antibody production in middle-aged mice requires phospholipase $A_{2} G 2 D$
}

\author{
Jian Zheng, ${ }^{1}$ David Meyerholz, ${ }^{2}$ Lok-Yin Roy Wong, ${ }^{1}$ Michael Gelb, ${ }^{3}$ Makoto Murakami, ${ }^{4}$ and Stanley Perlman \\ 'Department of Microbiology and Immunology and ${ }^{2}$ Pathology, University of lowa, lowa City, lowa, USA. ${ }^{3}$ Departments of Chemistry and Biochemistry, University of Washington, Seattle, Washington, USA. \\ ${ }^{4}$ Center for Disease Biology and Integrative Medicine, Graduate School of Medicine, The University of Tokyo, Tokyo, Japan.
}

\begin{abstract}
Worse outcomes occur in aged compared with young populations after infections with respiratory viruses, including pathogenic coronaviruses (SARS-CoV, MERS-CoV, and SARS-CoV-2), and are associated with a suboptimal lung milieu ("inflammaging"). We previously showed that a single inducible phospholipase, $P L A_{2} C 2 D$, is associated with a proresolving/antiinflammatory response in the lungs, and increases with age. Survival was increased in naive Pla2g2 $d^{-/-}$mice infected with SARS-CoV resulting from augmented respiratory dendritic cell ( $\mathrm{rDC}$ ) activation and enhanced priming of virus-specific T cells. Here, in contrast, we

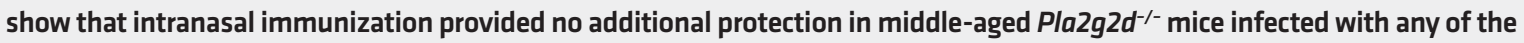
3 pathogenic human coronaviruses because virtually no virus-specific antibodies or follicular helper CD4+ $T$ (Tfh) cells were produced. Using MERS-CoV-infected mice, we found that these effects did not result from $\mathrm{T}$ or $\mathrm{B}$ cell intrinsic factors. Rather, they resulted from enhanced, and ultimately, pathogenic rDC activation, as manifested most prominently by enhanced IL-1 $\beta$ expression. Wild-type rDC transfer to $\mathrm{Pla} 2 \mathrm{~g} 2 \mathrm{~d}^{-/-}$mice in conjunction with partial IL-1 $\beta$ blockade reversed this defect and resulted in increased virus-specific antibody and Tfh responses. Together, these results indicate that PLA $\mathrm{C}_{2} \mathrm{D}$ has an unexpected role in the lungs, serving as an important modulator of $\mathrm{rDC}$ activation, with protective and pathogenic effects in respiratory coronavirus infections and immunization, respectively.
\end{abstract}

\section{Introduction}

Pathogenic human coronaviruses, which include severe acute respiratory syndrome-coronavirus (SARS-CoV), Middle East respiratory syndrome-CoV (MERS-CoV), and SARS-CoV-2, cause lethal pneumonia. COVID-19, caused by SARS-CoV-2, has reached pandemic proportions, with 158 million confirmed cases and 3.3 million deaths as of May 11, 2021 (1). SARS, MERS, and COVID-19 are most severe in aged individuals and those with underlying comorbidities (2-4). While SARS-CoV-2 has caused a pandemic, MERS-CoV has been identified by the WHO as a priority pathogen and by the Coalition for Epidemic Preparedness Innovation as a candidate for vaccine development because it also causes severe disease without therapeutic options and has epidemic potential (5).

Oxidative stress and chronic low-grade inflammation (inflammaging; refs. 6,7 ) occur in the lungs during aging, are associated with immune system dysfunction $(8,9)$, and result in less effective development of immune memory after vaccination or natural infection $(10,11)$. Inflammaging in the lungs is countered, in part, by age-dependent increased expression of a single inducible phospholipase with antiinflammatory/proresolving properties,

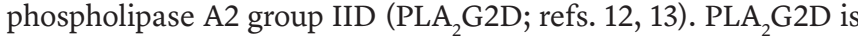
increased in lung dendritic cells (DCs) as mice age (13) and contributes to the production of several antiinflammatory lipid mediators (e.g., prostaglandin D2 $\left[\mathrm{PGD}_{2}\right], \mathrm{PGE}_{2}, \mathrm{PGF}_{2 \alpha}$, and thromboxane

Conflict of interest: The authors have declared that no conflict of interest exists. Copyright: (5) 2021, American Society for Clinical Investigation.

Submitted: December 28, 2020; Accepted: April 14, 2021; Published: June 1, 2021.

Reference information: J Clin Invest. 2021;131(11):e147201.

https://doi.org/10.1172/JCl147201.
B2; refs. 12-14). However, we found that these antiinflammatory responses contributed to a delayed immune response and, subsequently, delayed kinetics of virus clearance after infection with SARS-CoV, which rapidly replicates to high titers in the lungs (15). Decreased age-related survival in SARS-CoV-infected mice was largely reversed in Pla $2 \mathrm{~g} 2 \mathrm{~d}^{-/-}$mice as a consequence of enhanced respiratory dendritic cell (rDC) migration from the lungs to draining lymph nodes and subsequent augmented virus-specific $\mathrm{T}$ cell responses (13). Since vaccination efficacy is lower in aged compared with young mice, we reasoned that the absence of PLA ${ }_{2}$ G2D would also enhance memory $\mathrm{T}$ cell and antibody responses after intranasal (i.n.) immunization with sublethal virus infection or with other immunogens, thereby increasing vaccine efficacy.

To assess this possibility, we immunized and infected mice with MERS-CoV, SARS-CoV, or SARS-CoV-2. Most of the studies described below study MERS-CoV-infected mice. Mice were immunized with a sublethal dose of MERS-CoV (sublethal infection) or with a replication-incompetent alphavirus replicon particle expressing the spike protein or nucleocapsid of MERSCoV (VRP-MERS-S, VRP-MERS-N). Mice are normally resistant to infection with MERS-CoV, but are rendered susceptible to the virus if the human receptor human dipeptidyl peptidase-4 (hDPP4) is present. For this purpose, we generated mice knockedin for $h D P P 4$ and then further adapted the virus to mice by serial passage through these mice (16). We then produced $h D P P 4$ mice lacking Pla2g2d expression ( $h D P P 4-P l a 2 g 2 d^{-/-}$mice) by crossing the $h D P P 4$ mice with Pla $2 g 2 d^{-/-}$mice. To study SARS, we directly infected Pla2 $\mathrm{g}_{2} \mathrm{~d}^{-/-}$mice with mouse-adapted SARS-CoV, as previously described (13). Mice are naturally resistant to SARS-CoV-2, but become susceptible if the human receptor (human angioten- 
sin converting enzyme-2 [hACE2]) is provided using an adenovirus encoding hACE2 (Ad5-hACE2; refs. 17, 18). Use of Ad5-hACE2 transduction allowed for infection of Pla2g2 $\mathrm{d}^{-/-}$and Pla2g2 $2 \mathrm{~d}^{+/+}$ mice with human strains of SARS-CoV-2.

Here, in contrast to results observed in mice with acute respiratory $\mathrm{CoV}$ infection, we found that i.n. immunization of middle-aged (5-8 months old) but not young (8 weeks old) Pla2g2 $\mathrm{d}^{-/}$ mice resulted in no improvement in outcomes after MERS-CoV challenge because virtually no virus-specific antibody was produced. Similar results were found in mice infected with SARS$\mathrm{CoV}$ or SARS-CoV-2. Antibody deficiency resulted from a nearly complete absence of a follicular helper $\mathrm{CD} 4^{+} \mathrm{T}$ cell (Tfh) response. As in the acute infection, activation of rDCs was enhanced in Pla2g $2 d^{-/}$mice, but in this case, it resulted in poor Tfh cell and virus-specific antibody production.

\section{Results}

$P L A_{2} G 2 D$ deficiency impairs protection in middle-aged but not young mice after MERS-CoV immunization and challenge. To address the role of PLA $\mathrm{G}_{2} \mathrm{D}$ in memory immune responses, we immunized middle-aged (5-6 month old) $h D P P 4$ and $h D P P 4-P l a 2 g 2 d^{-/-}$mice with a sublethal dose of virus (Figure $1, \mathrm{~A}^{-} \mathrm{C}$ ) or VRP-MERS-S (Figure 1, D-I) and then challenged them with a lethal dose of MERS-CoV. Prior to analyzing these mice, we found that acute MERS-CoV infection of naive middle-aged Pla $2 \mathrm{~g}^{2 \mathrm{~d}^{-/-}}$mice resulted in more rapid kinetics of virus clearance (Figure 1, B and E), decreased pathological damage (Supplemental Figure 1, A and B; supplemental material available online with this article; https:// doi.org/10.1172/JCI147201DS1), and diminished morbidity and mortality (Figure 1, C and F) compared with infected hDPP4 mice, consistent with studies of SARS-CoV (13). In contrast, while immunization with a sublethal dose of MERS-CoV protected middle-aged hDPP4 mice from weight loss and death and enhanced the kinetics of virus clearance, it did not increase survival of middle-aged $h D P P 4-P l a 2 g 2 d^{-/-}$mice (Figure 1, B and C).

A lack of protection was not confined to sublethal infection because similar results were observed in middle-aged $h D P P 4$ and hDPP4-Pla2g2 $d^{-/-}$mice that were i.n. primed and boosted with VRP-MERS-S $(19,20)$ and then challenged with a lethal dose $(750$ pfu) of MERS-CoV (Figure 1D). Immunization with VRPs expressing exogenous proteins has been shown to be protective in several virus infections (21). While VRP-MERS-S immunization augmented the kinetics of virus clearance and protected middle-aged hDPP 4 mice from death, it had no effect on virus clearance (Figure $1 \mathrm{E}$ ) or survival (Figure $1 \mathrm{~F}$ ) of middle-aged hDPP4-Pla $2 \mathrm{~g}^{-/-}$ mice after MERS-CoV challenge. Consistently, increased lung damage was found in infected immunized middle-aged hDPP4Pla2g $2 d^{-/-}$compared with hDPP4 mice (Supplemental Figure 1, A and B). Pla $2 g 2 d$ increases as mice age (13). Consistent with these results and in contrast to results obtained with middle-aged mice, immunization completely protected young $h D P P 4$ and $h D P P 4-$

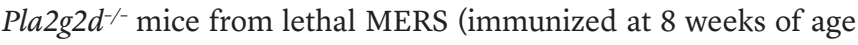
and challenged 70 days later) (Figure $1 G$ ).

Similar results were obtained in mice infected with SARSCoV-2 or SARS-CoV. For SARS-CoV-2 immunization, Ad5hACE2-transduced mice were infected with $10^{5}$ pfu SARS-CoV-2 since the infection is nonlethal. For challenge, mice were again transduced with Ad5-hACE2 and challenged with the same dose of virus (Supplemental Figure 2A). Of note, hACE2 expression was not diminished after the second transduction with Ad5-hACE2 (Supplemental Figure 2B). In agreement with previous reports (17, 18), infected Ad5-hACE2-transduced mice lost weight but all survived. SARS-CoV-2 infection caused less weight loss in Ad5-hACE2-transduced naive Pla2g2 $d^{-/}$compared with naive Pla2g2 $d^{+/+}$(WT) mice. However, prior immunization protected Ad5-hACE2-transduced WT mice from weight loss and enhanced the kinetics of virus clearance after a second challenge, but had no ameliorating effect on viral clearance or clinical disease in Pla2g2d $\mathrm{d}^{-/}$mice (Supplemental Figure 2, C and D). For SARSCoV, we immunized 5-month-old mice i.n. with VRP-SARS-S and challenged them with a lethal dose of SARS-CoV 2 months later (Supplemental Figure 2F). In the absence of immunization, sur-

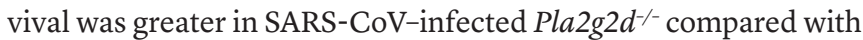
Pla2g $2 d^{+/+}$mice (Supplemental Figure $2 \mathrm{G}$ ), in agreement with published results (13). As in MERS-CoV-infected mice, VRP-SARS-S immunization completely protected SARS-CoV-infected WT mice from severe disease but had no effect on clinical outcomes in infected Pla2g2 $\mathrm{d}^{-/-}$mice (Supplemental Figure 2G).

To assess whether the defect in memory response generation was unique to i.n. inoculation, we immunized middle-aged mice intraperitoneally (i.p.) with VRP-MERS-S (Figure 1H). Immunization of either Pla $2 \mathrm{~g}^{2} \mathrm{~d}^{-/-}$or WT mice was equally protective after i.n. challenge with a lethal dose of virus (Figure 1I), demonstrating that the effects of the absence of PLA ${ }_{2}$ G2D were confined to the respiratory tract.

Intranasally immunized middle-aged Pla2g2 $d^{-/}$mice mount minimal CoV-specific antibody responses after virus challenge. Virus-specific neutralizing antibodies are critical for protection against rechallenge with homologous virus (22), so next we assessed whether differences in virus-specific antibody responses in hDPP4 and hDPP4-Pla2g2 $d^{-/-}$mice after i.n. or i.p. immunization and MERS-CoV challenge contributed to the observed phenotypes. Unexpectedly, we detected virtually no serum anti-virus neutralizing $\left(\mathrm{PRNT}_{50}\right)$ antibodies after cognate virus challenge of middle-aged $h D P P 4-P l a 2 g 2 d^{-/-}$mice that had been i.n. immunized with a sublethal dose of MERS-CoV (Figure 2A) or VRP-MERS-S (Figure 2C). A similar lack of virus-specific antibody response was observed in middle-aged mice immunized and challenged with SARS-CoV-2 (Supplemental Figure 2E) or SARS-CoV (Supplemental Figure 2H).

Equivalent $\mathrm{PRNT}_{50}$ responses were detected in young $h D P P 4$ and $h D P P 4-P l a 2 g 2 d^{-/-}$mice (primed at 8 weeks) immunized with a sublethal dose of MERS-CoV (Figure 2B) or VRP-MERS-S (Figure 2D), and challenged with cognate virus. Of note, virus clearance was more rapid in both young and middle-aged $h D P P 4-P l a 2 g 2 d^{-/-}$ compared with hDPP4 mice, respectively, after immunization in the absence of challenge (Supplemental Figure 3), but antibody production was only defective in the middle-aged mice. These results indicate that more rapid virus clearance was not a major factor responsible for impaired virus-specific antibody production in the older group. Consistent with the clinical data shown in Figure 1I, i.p. immunization of middle-aged $h D P P 4-P l a 2 g 2 d^{-/-}$and hDPP4 mice resulted in equivalent production of virus-specific antibodies (Figure 2E). These results indicate that there is an 
A

Sublethal dose of Lethal dose of MERS-COV or PBS MERS-CoV

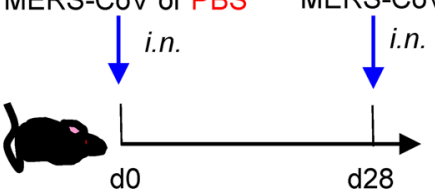

$6 \mathrm{~m} h D P P 4$ or hDPP4Pla2g2d/- mice
B

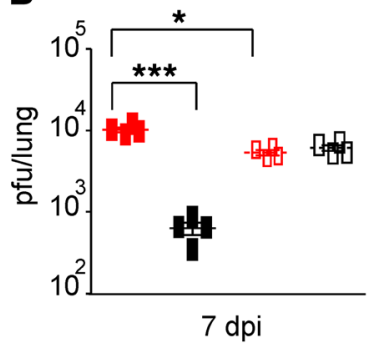

C - Naive hDPP4 - - Naive hDPP4-Pla2g2d-

- Immunized hDPP4 - - Immunized hDPP4-Pla2g2d-

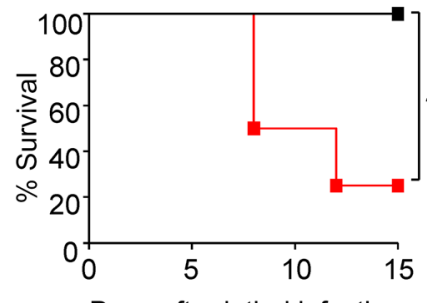

Days after lethal infection

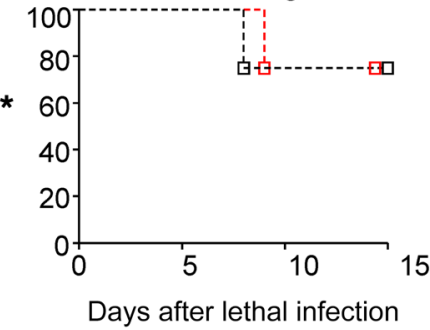

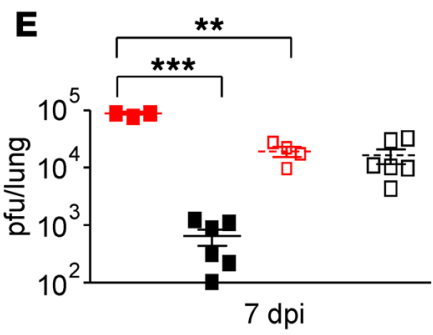

Naive hDPP4
Immunized $h D P P 4$
$-\square \cdot$ Naive $h D P P 4-P l a 2 g 2 d^{-}$
$-\square \cdot$ Immunized $h D P P 4-P l a 2 g 2 d^{--}$

$8 \mathrm{wk} / 5 \mathrm{~m} h D P P 4$ or

do $\quad$ d28

d70

hDPP4-Pla2g2d-1-

mice

$\mathbf{F}$

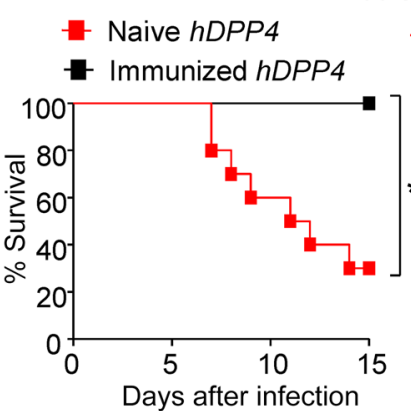

Middle-aged mice

$-\boxminus$. Naive hDPP4-Pla2g2d ${ }^{-1}$

$\square \cdot$ Immunized $h D P P 4-P l a 2 g 2 d^{-1-}$

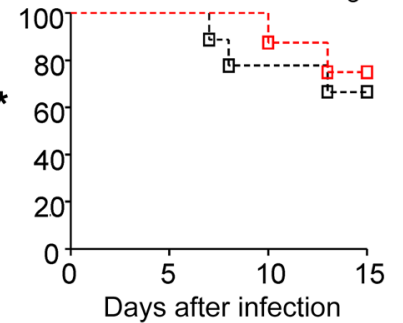

G

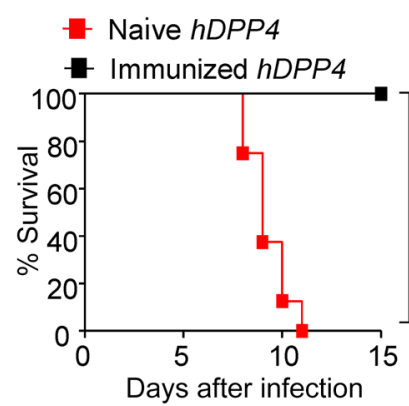

Young mice

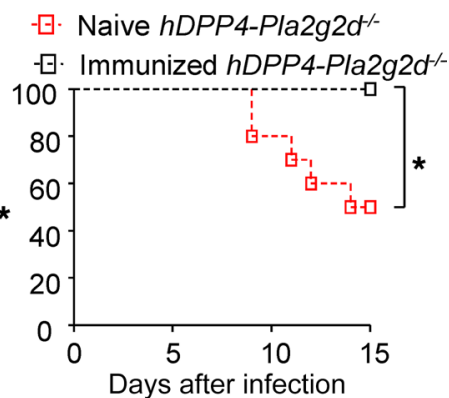

H

i.p. immunization

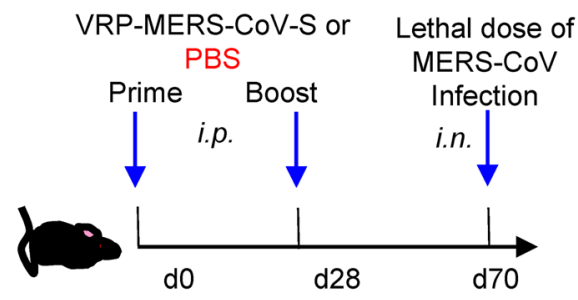

$5 \mathrm{~m} \mathrm{hDPP4}$ or hDPP4-Pla2g2d--

mice
I Naive hDPP4

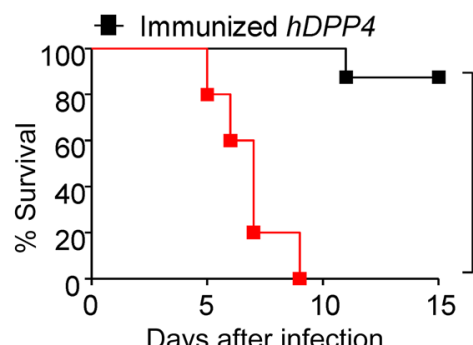

Days after infection $-\boxminus \cdot$ Naive hDPP4-Pla2g2d ${ }^{-/}$

$-\square$. Immunized hDPP4-Pla2g2 $d^{-1-}$

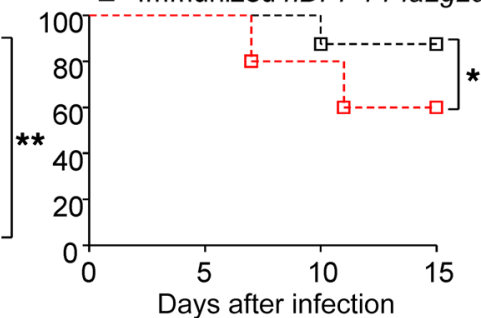

Figure 1. PLA 2 G2D deficiency impairs immunization-induced protection in middle-aged mice challenged with MERS-CoV. (A) Protocol for MERS-CoV sublethal infection and challenge. Six-month-old hDPP4 or hDPP4-Pla2g2d-1- mice were immunized with a sublethal dose (100 pfu) of MERS-CoV or PBS i.n. on day 0, followed by i.n. infection with a lethal dose (750 pfu) of MERS-CoV on day 28. Virus titers in lungs at $7 \mathrm{dpi}(n=6)(\mathbf{B})$ and survival $(n=8-10$ / group) (C) of naive and immunized 6-month-old hDPP4 or hDPP4-Pla2g2d-/- mice are shown. (D) Protocol for VRP-MERS-S immunization and MERSCoV infection. 8-week-old (young) or 5-month-old (middle-aged) hDPP4 or hDPP4-Pla2g2d-/- mice were treated with VRP-MERS-S or PBS i.n. on day 0 and day 28 , followed by a lethal dose ( 250 pfu for young mice and 750 pfu for middle-aged mice) of MERS-CoV i.n. on day 70 . (E) Virus titers in lungs of middle-aged $h D P P 4$ or $h D P P 4-P l a 2 g 2 d^{-1-}$ mice at $7 \mathrm{dpi}$ are shown, $n=6 /$ group. (F) Survival of infected middle-aged mice, $n=8$ to $10 /$ group. (C) Survival of infected young (8-week-old) mice, $n=8$ to 10/group. (H) Protocol for i.p. VRP immunization. Five-month-old $h D P P 4$ or $h D P P 4-P l a 2 g 2 d^{-/-}$mice were immunized with VRP-MERS-S or PBS i.n. on day 0 and day 28 , followed by infection with a lethal dose (250 pfu for young mice and 750 pfu for middle-aged mice) of MERS-CoV i.n. on day 70. (I) Survival of i.p. immunized and infected $h D P P 4$ or $h D P P 4-P l a 2 g 2 d^{-1-}$ mice is shown, $n=20 /$ group. (B and E) Data are shown as mean \pm SEM and analyzed using 1-way ANOVA. (C, F, G, and I) Survival rates of groups were analyzed using Kaplan-Meier log-rank survival tests. All data are representative of 3 independent experiments. ${ }^{*} P<0.05$, ${ }^{* *} P<0.01,{ }^{* * *} P<0.001$. 
A

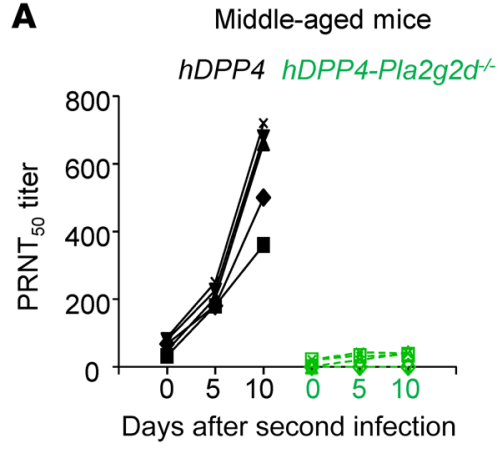

B Young mice

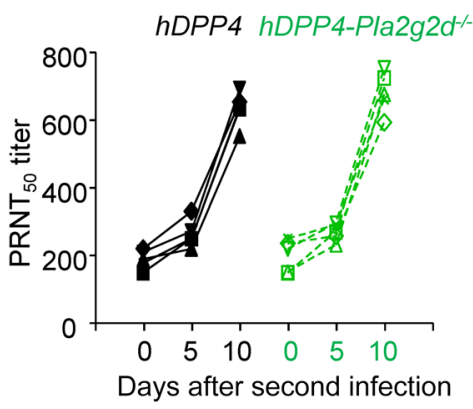

Immunization with VRP-MERS-S

C Middle-aged mice-i.n.

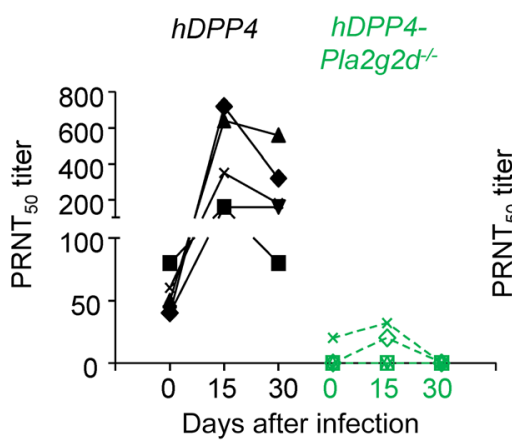

$\mathbf{F}$

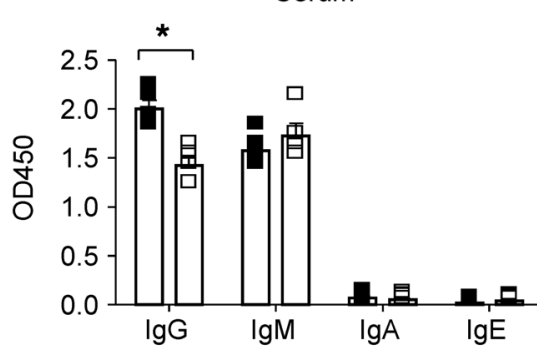

D

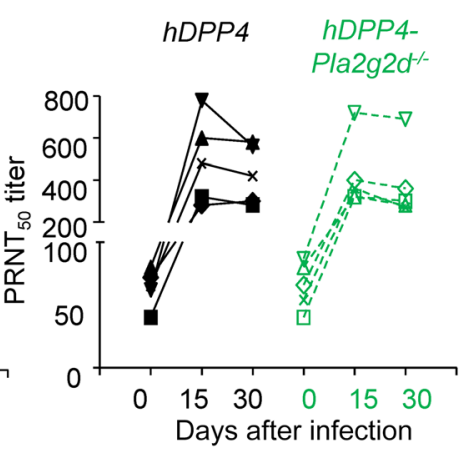

E

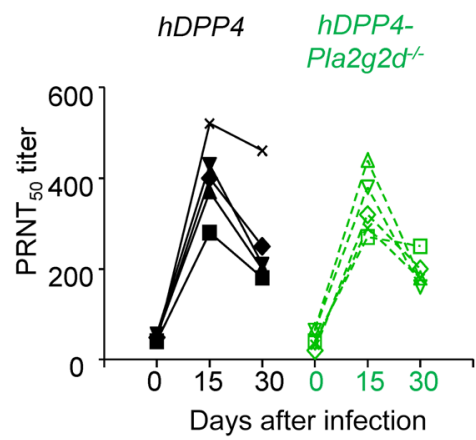

Middle-aged mice-i.p

hDPP4 hDPP4

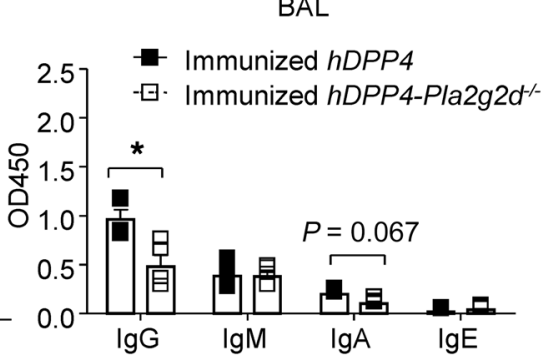

Figure 2. Impaired vaccine efficacy in MERS-CoV-challenged middle-aged Pla2g2d-1- mice results from a deficiency in neutralizing antibody production. Middle-aged (A, C, E, and F) or young (B and $\mathbf{D}) h D P P 4$ or $h D P P 4-P l a 2 g 2 d^{-1-}$ mice were immunized with a sublethal dose of MERS-CoV (A and B) or VRP-MERS-S (C-F) and then challenged with lethal dose of MERS-CoV as described in Figure 1. Virus-specific serum neutralizing antibody titers (47) were measured by PRNT $_{50}$ assay at indicated days after challenge as described in Methods, $n=5 /$ group. (A-E) Each data point represents 1 animal. (F)

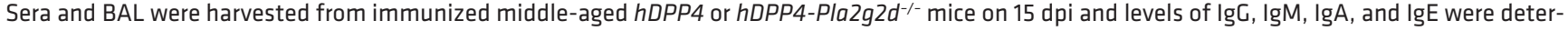
mined by ELISA as described in Methods, $n=6$ to 12/group. Representative data of 3 independent experiments are shown as mean \pm SEM and analyzed using Student's $t$ test. ${ }^{*} P<0.05$.

essential role for PLA ${ }_{2}$ G2D expression in virus-specific antibody responses in i.n. immunized and challenged middle-aged mice.

Impairment of CoV-specific antibody responses in middle-aged Pla2g2d- mice after immunization is not B cell intrinsic. The absence of a virus-specific neutralizing antibody response in immunized middle-aged Pla2g $2 d^{-/}$mice after challenge could reflect a generalized defect in antibody production, an inability to mount a virus-specific response after i.n. immunization, or normal responses to the vaccine but rapid waning of virus-specific antibodies before challenge. To distinguish among these mechanisms, we examined the B cell compartment in immunized $h D P P 4$ and $h$ DPP4-Pla2g2 $d^{-/-}$mice before challenge. We i.n. immunized 5-month-old hDPP4 and hDPP4-Pla2g2d $d^{-/}$mice with VRP-
MERS-S and, additionally, VRP-MERS-N (expressing the nucleocapsid [N] protein, Figure $3 \mathrm{~A}$ ) for the reasons described below. We detected only modest differences in total IgG in the sera and bronchoalveolar lavage fluid (BAL) (Figure 2F and Supplemental Figure 4A). However, there was a nearly complete absence of serum MERS-CoV-specific antibodies measured using ELISPOT assays or PRNT ${ }_{50}$ in hDPP4-Pla2g2 $d^{-/}$mice after i.n. priming and boosting (in the absence of challenge) (Figure 3B and Supplemental Figure $4 \mathrm{~B})$. Further, we detected an increase in numbers of germinal center B cells $\left(\mathrm{CD} 3{ }^{-} \mathrm{B} 22 \mathrm{O}^{+} \mathrm{CD} 27^{-} \mathrm{CD} 138^{-} \mathrm{CD} 23^{+} \mathrm{GL} 7^{+}\right)$in the DLNs of i.n. immunized $h D D P 4$ but not $h D P P 4-P l a 2 g 2 d^{-/}$mice (Figure 3 , $\mathrm{C}$ and $\mathrm{D})$. There was also an absence of bronchus-associated lymphoid tissue (BALT, ectopic germinal center) in hDPP4-Pla2g2d $\mathrm{d}^{-/}$ 
A

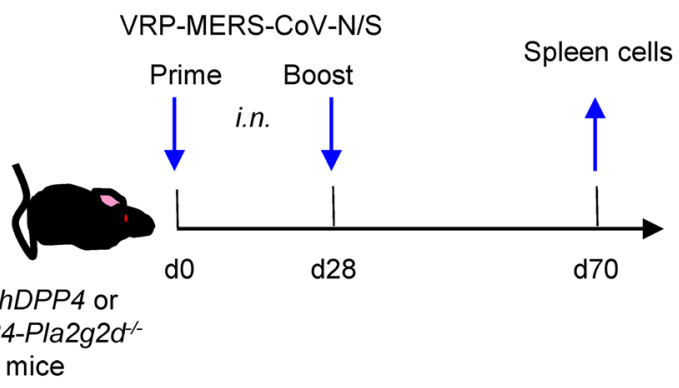

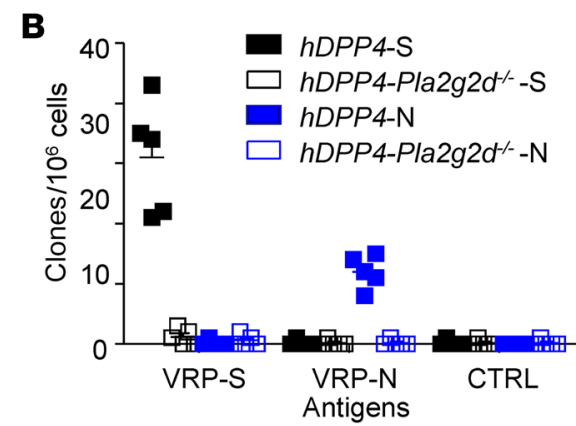

C

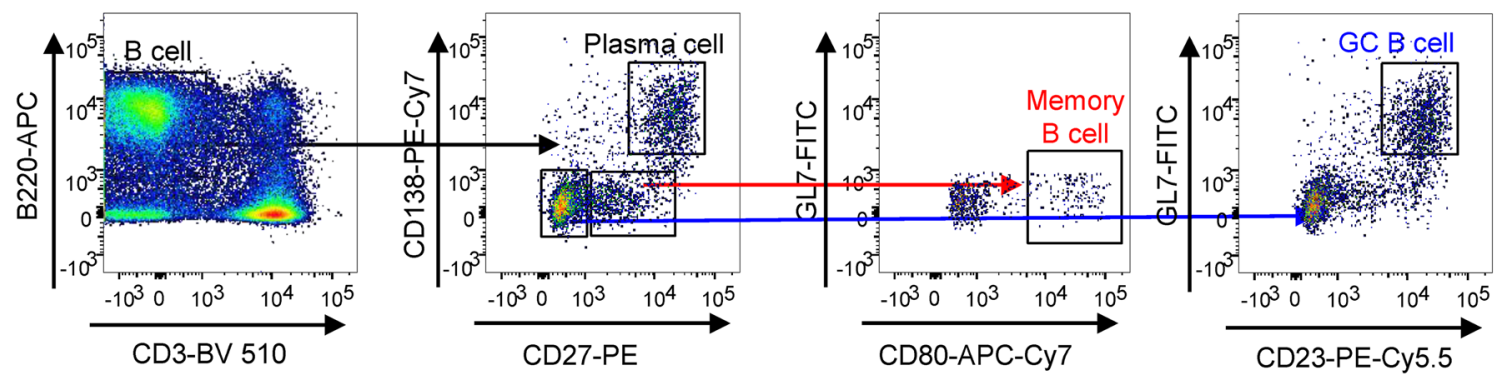

D
Naive Pla2g2d
- Immunized Pla2g2d
- Im+/
- Naive Pla2g2d ${ }^{-}$
-曰. Immunized Pla2g2d-
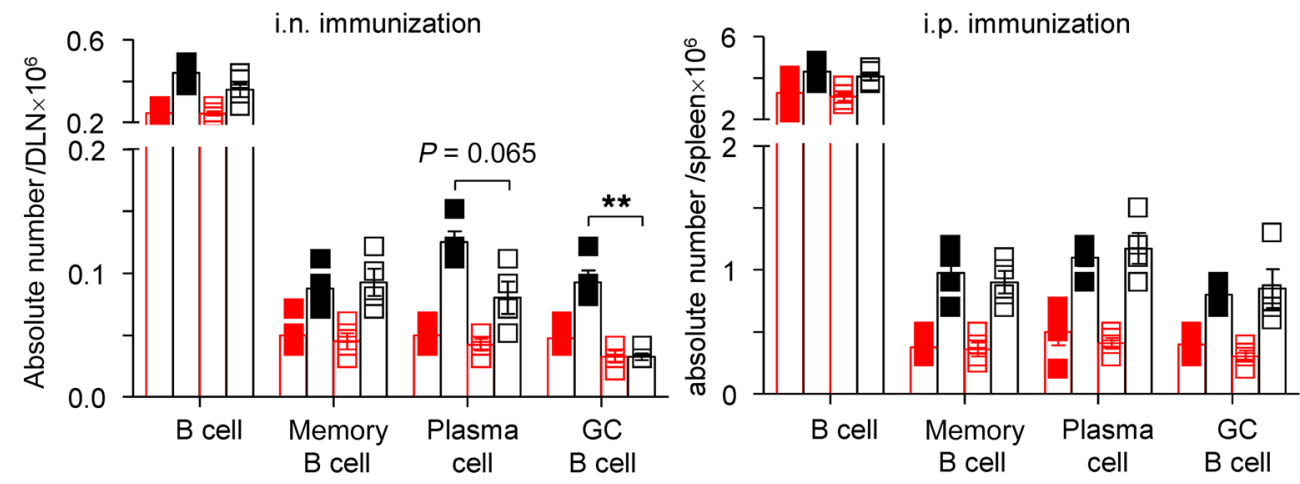

E

hDPP4

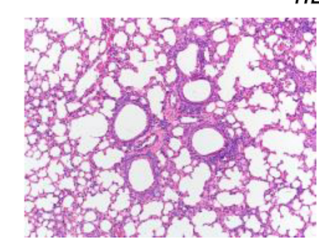

$10 \times$

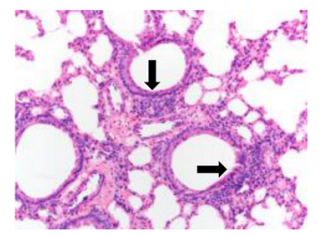

$20 x$

Day 30 after boost

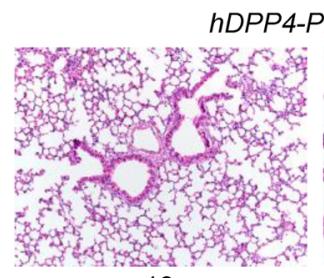

$10 x$

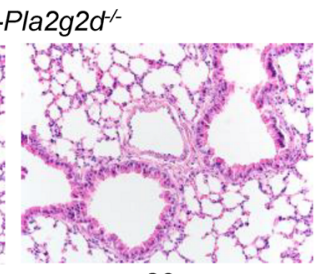

$20 \times$

Figure 3. Immunized middle-aged hDPP4-Pla2g2d-- mice showed impaired virus-specific antibody production and germinal center B cell expansion. (A) Protocol for VRP immunization and sample collection. Five-month-old hDPP4 or hDPP4-Pla2g2d $d^{-1-}$ mice were immunized with VRP-MERS-S/VRPMERS-N, or PBS i.n. on day 0 and day 28. (B) Virus-specific antibody-producing cells in the spleens of VRP-MERS-S or VRP-MERS-N immunized mice were identified using ELISPOT assays as described in Methods, $n=5 /$ group. (C) Gating strategy for detecting B cell subsets in DLN: total B cell (CD3-B220 ${ }^{+}$), memory B cell (CD3-B220+CD27+CD80+CL7-), plasma cell (CD3-B220+CD27+CD138+), germinal center B cell (CC B, CD3-B222+CD27-CD23+CL7+). (D) Numbers of total $B$ cell and $B$ cell subsets in naive and immunized mice, $n=5 /$ group, were determined as described in Methods. (E) Bronchial-associated lymphoid-like tissue in lungs of $h D P P 4$ and $h D P P 4-P l a 2 g 2 d^{-/-}$mice at day 30 after boosting with VRP-MERS-S (indicated with arrow). (B and D) Data are shown as mean \pm SEM and are representative of 3 independent experiments. Data shown in $\mathbf{D}$ were compared using 1-way ANOVA. ${ }^{* *} P<0.01$.

compared with $h D P P 4$ lungs after immunization (Figure 3E). In contrast, neutralizing antibody production (Supplemental Figure $4 \mathrm{C})$ and numbers of germinal center B cells (Figure 3D) were equivalent after i.p. immunization of $h D P P 4$ and $h D P P 4-P l a 2 g 2 d^{-1-}$ mice with VRP-MERS-S. Thus, impaired CoV-specific antibody production was observed after i.n. but not systemic immunization and did not result from a global B cell-intrinsic defect.

Absence of follicular helper $C D 4^{+} T$ cell (Tfh) response in intranasally immunized/infected middle-aged Pla2g2d-- mice. Generation of an optimal virus-specific antibody response requires a robust 
A

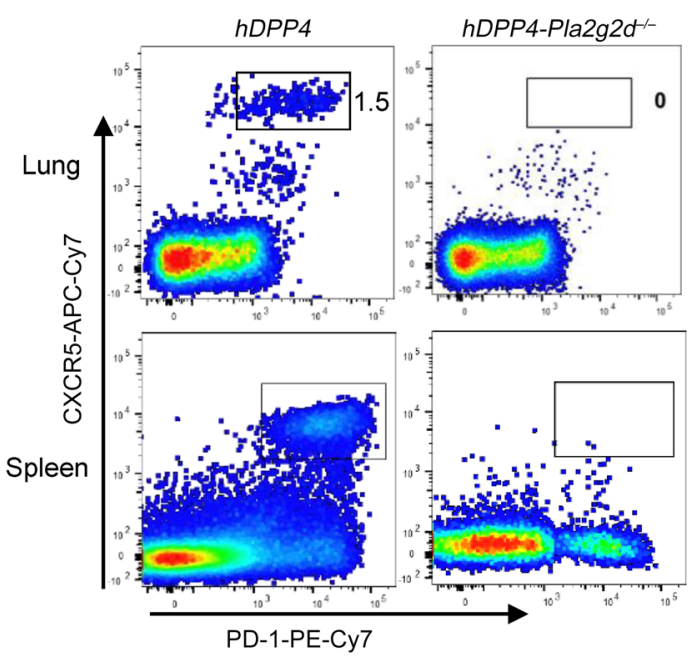

B i.n. immunization

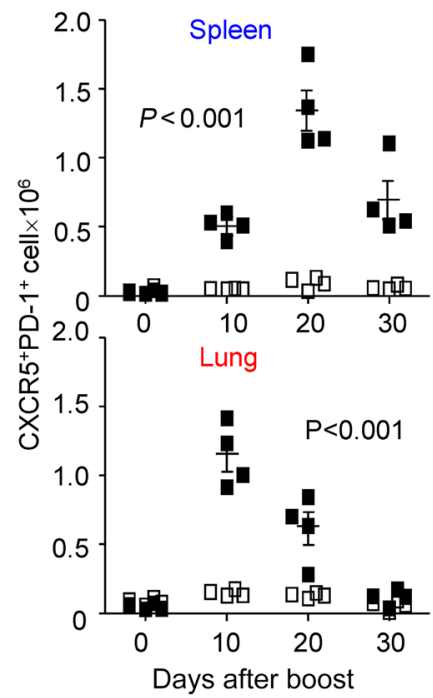

C i.p. immunization

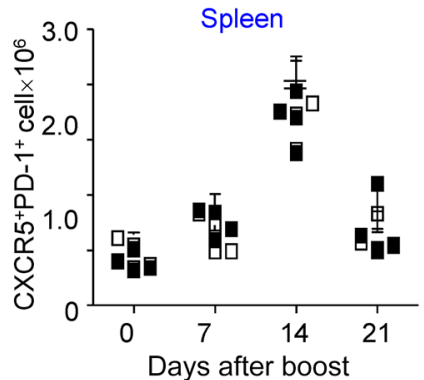

- Immunized hDPP4

$\because \cdot$ Immunized hDPP4-Pla2g2dt-

D
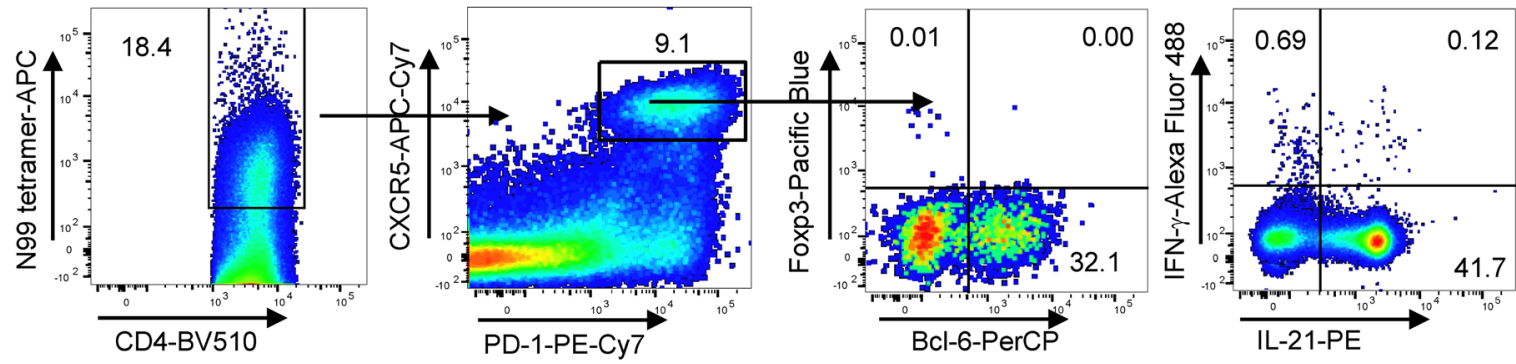

Immunized hDPP4 -曰- Immunized hDPP4-Pla2g2d-1-

E $\mathrm{N} 99+\mathrm{CD} 4$

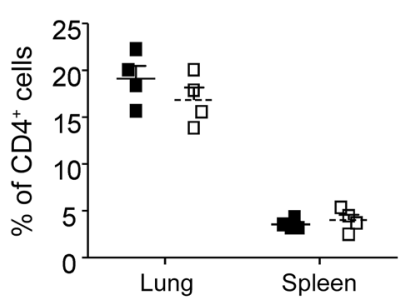

G

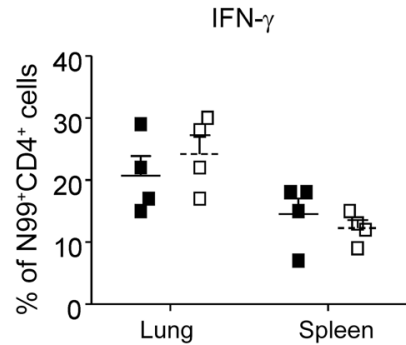

Tfh

$\left(\mathrm{CXCR5} 5^{+} \mathrm{PD}-1^{+}\right)$

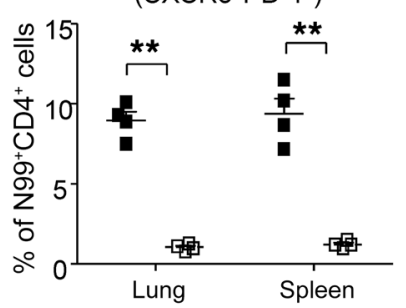

$\mathbf{F}$

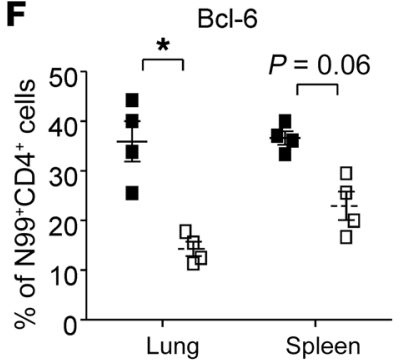

Foxp3

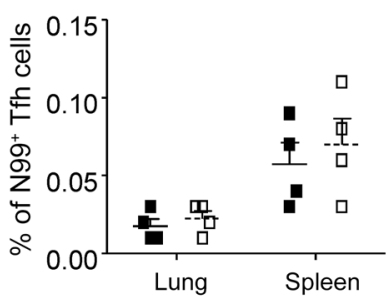

Figure 4. Middle-aged $h D P P 4-P l a 2 g \mathbf{2 d}^{-/-}$mice show impaired Tfh cell development after immunization and challenge. (A) Five-month-old $h D P P 4$ or hDPP4-Pla2g2 $d^{-1-}$ mice were immunized with VRP-MERS-S i.n. on day 0 and day 28 . Flow plots of PD- $1^{+} \mathrm{CXCR} 5^{+}$(Tfh) CD4 $4^{+} \mathrm{T}$ cells in lungs and spleens of mice sacrificed on day 35 after priming are shown. (B and $\mathbf{C}$ ) The number of total Tfh cells in lungs and spleens of mice at indicated days after i.n. immunization (B) or i.p. immunization (C), $n=4$ /group at each time point. (D-C) Five-month-old $h D P P 4$ or hDPP4-Pla2g2d $d^{-/-}$mice were immunized with VRP-MERS-N i.n. on day 0 and day 28. (D) Gating strategy (using samples from hDPP4 mice). (E and F) N99+ CD4+ $4^{+}$cells were detected using I-A ${ }^{\mathrm{b}} / \mathrm{N} 99$ tetramers. Percentage of total N99+ CD4 in total CD4+ $\mathrm{T}$ cells, Tfh cells (CXCR5+PD-1+) in N99+ $\mathrm{CD} 4^{+} \mathrm{T}$ cells (E), and Bcl-6+ cells in N99+ CD4+ $\mathrm{T}$ cells and Foxp3 ${ }^{+}$cells in CD4 ${ }^{+}$Tfh cells (CXCR5 ${ }^{+} \mathrm{PD}-1^{+}$CD4 $4^{+} \mathrm{T}$ cells) (F), $n=4$ /group. (C) Expression of IFN- $\gamma$, IL-21, IL-2, and TNF by lung and splenic N99+ CD4 ${ }^{+} \mathrm{T}$ cells was determined after stimulation with a MERS-CoV N protein peptide pool as described in Methods, $n=4$ /group. (B, C, and E-C) Data are shown as mean \pm SEM and are representative of $\mathbf{3}$ independent experiments. Data shown in $\mathbf{B}$ and $\mathbf{C}$ were compared using multiple regression analysis. Data shown in $\mathbf{E}-\mathbf{C}$ were analyzed using Student's $t$ test. ${ }^{*} P<0.05,{ }^{* *} P<0.01$. 
A $\quad$ - Immunized $h D P P 4$

$-\square \cdot$ Immunized hDPP4-Pla2g2d ${ }^{--}$
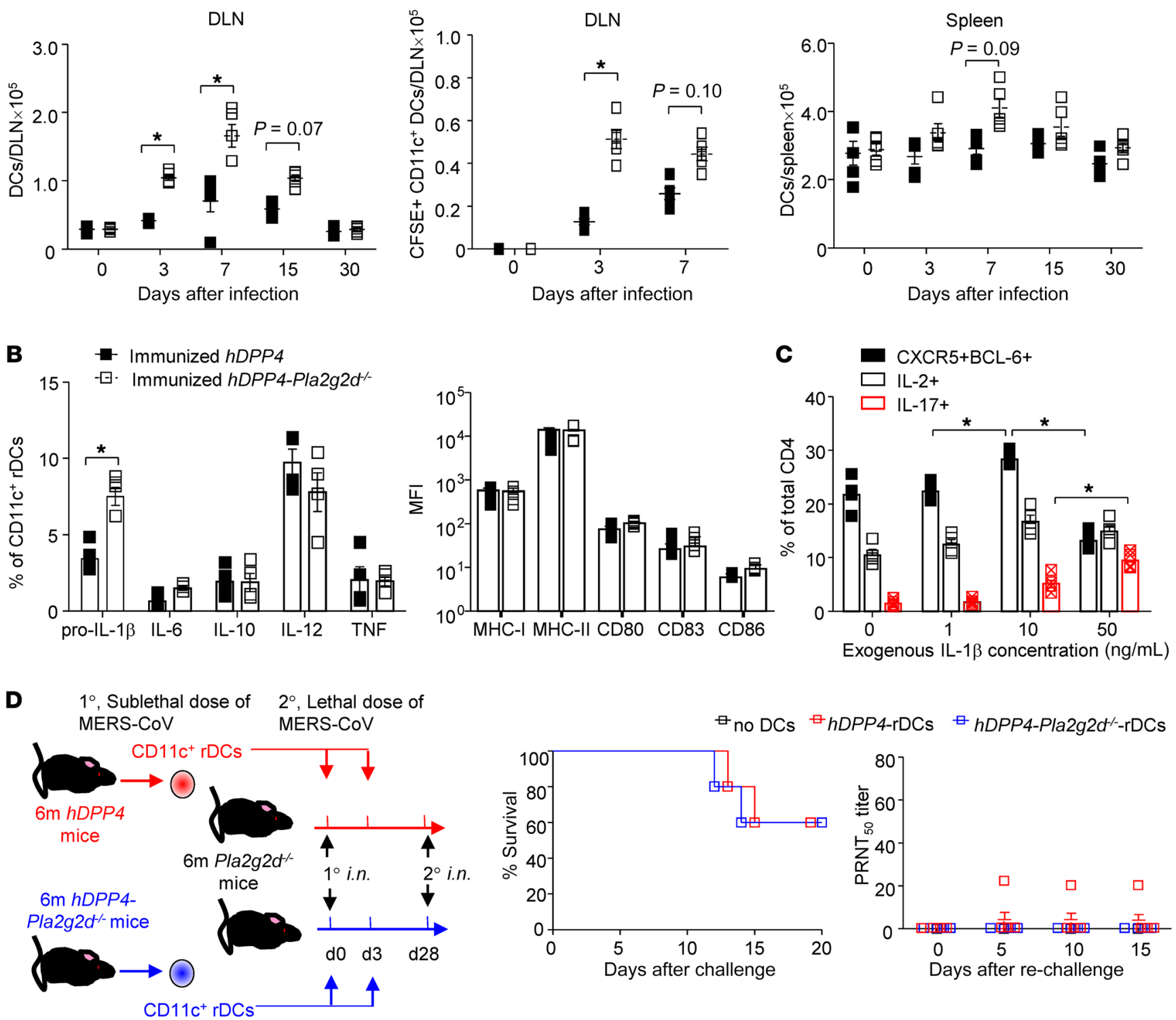

Figure 5. Immunization induces increased accumulation of and IL-1 $\beta$ expression by CD11c ${ }^{+}$rDCs in DLNs of hDPP4-Pla2g2d $d^{-/-}$mice. Five-month-old hDPP4 and hDPP4-Pla2g2d ${ }^{-1-}$ mice were immunized with VRP-MERS-S or PBS i.n. on day 0 and day 28 , followed by a lethal dose (750 pfu) of MERS-CoV i.n. on day 70. Mice were treated intranasally with CFSE at day 0 after infection to track the migration of lung DCs. (A) The number of DCs in lung DLNs or spleens of VRP-MERS-S-immunized and infected middle-aged $h D P P 4$ or $h D P P 4-P l a 2 g 2 d^{-/-}$mice was determined by flow cytometry at the indicated dpi, $n$ = 4/group. (B) Expression of cytokines (pro-IL-1 $\beta$, IL-6, IL-10, IL-12, and TNF), MHC molecules, and costimulatory molecules (CD80, CD83, CD86) by lung and DLN DCs harvested from immunized hDPP4 or hDPP4-Pla $2 \mathrm{~g}^{2 \mathrm{~d}^{-/}}$mice is shown. Data are shown as mean \pm SEM and are representative of 3 independent experiments, $n=4$ /group. (C) An in vitro Tfh cell differentiation culture system was established as described in Methods. Percentage of CXCR5 ${ }^{+} B c l-6^{+}$, $\mathrm{IL}-2^{+}$, and IL-17+ $\mathrm{CD} 4^{+} T$ cells at day 4 of culture with different concentrations of exogenous IL-1 $\beta$ is shown, $n=5 /$ group. (D) DC adoptive transfer was carried out as shown in protocol. The survival of mice and production of neutralizing antibody were determined after challenge, $n=5 / \mathrm{group}$. (A-D) Data are shown as mean \pm SEM and are representative of 3 independent experiments. Data shown in $\mathbf{A}$ and $\mathbf{B}$ were analyzed using Student's $t$ test, while the percentages of $\mathrm{CXCR5}{ }^{+} \mathrm{Bcl}-6^{+}, \mathrm{IL}-2^{+}$, and IL-17+ $\mathrm{CD}^{+} \mathrm{T}$ cells shown in $\mathrm{C}$ were analyzed in an IL-1 $\beta$ dose-dependent manner using 1 -way ANOVA. ${ }^{*} P<0.05$.

Tfh response $(23,24)$. Tfh cells generally express surface markers CXCR5 and PD-1, the transcription factor Bcl-6, and IL-21 (2527). Examination of $\mathrm{T}$ cells isolated from the lungs of mice immunized with VRP-MERS-S revealed the nearly complete absence of $\mathrm{CD}^{+}{ }^{+} \mathrm{T}$ cells expressing CXCR5/PD-1 (Figure $4, \mathrm{~A}$ and B). Additionally, Tfh cells were almost completely absent from the lungs and spleens of mice immunized with SARS-CoV-2 (Ad5-hACE- transduced) or VRP-SARS-S (Supplemental Figure 5, A and B). As expected, Th cells were detected in equivalent numbers in young hDPP4 and hDPP4-Pla2g2d $d^{-/}$mice (Supplemental Figure 5C) or after i.p. immunization (Figure 4C).

We then assessed the CoV-specific Tfh response in immunized mice in the absence of challenge. The MERS-CoV N protein encodes an $\mathrm{I}^{\mathrm{b}} \mathrm{A}^{\mathrm{b}}$-restricted $\mathrm{CD} 4^{+} \mathrm{T}$ cell epitope $\mathrm{N} 99$, facilitating 
A

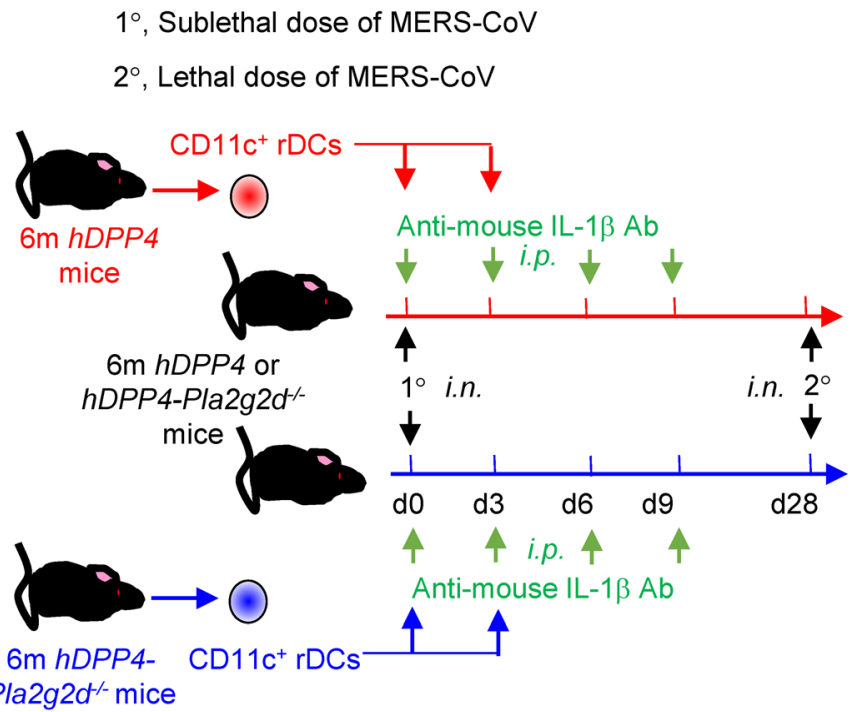

- $h D P P 4$ mice immunization alone

hDPP4-Pla2g2d/- mice

* Immunization alone

* Immunization+hDPP4-rDCs+ $\alpha-\mathrm{IL}-1 \beta$

\# Immunization $+h D P P 4-P l a 2 g 2 d^{-\alpha-r D C s}+\alpha-I L-1 \beta$

B

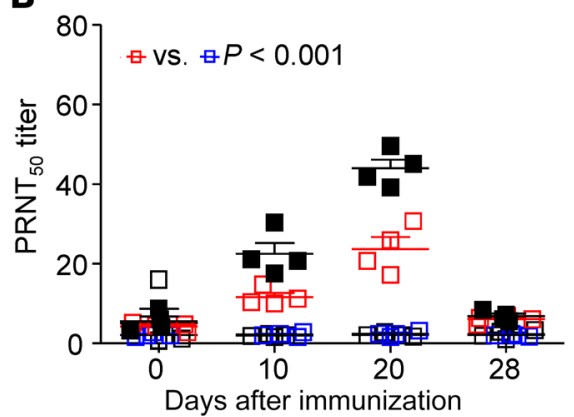

C

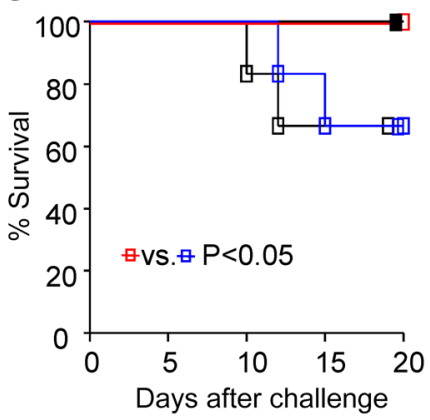

D

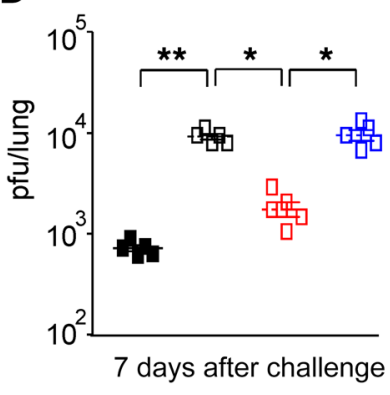

E

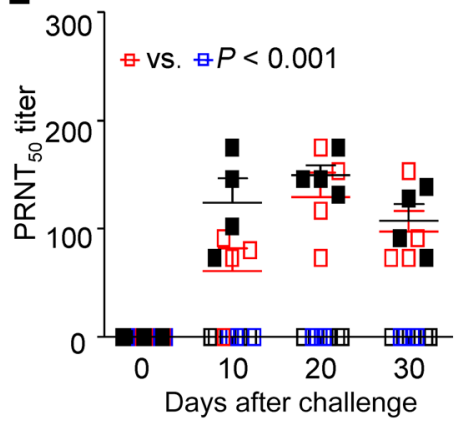

$\mathbf{F}$

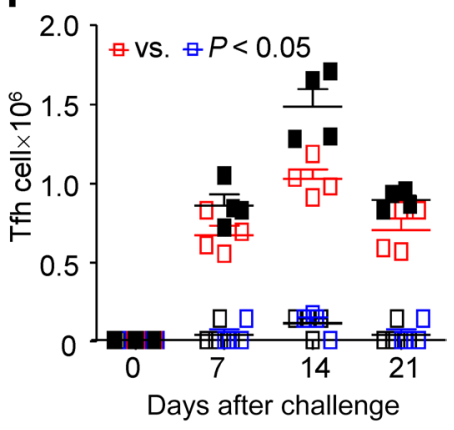

Figure 6. Adoptive transfer of hDPP4 CD11c ${ }^{+}$rDCs and low-dose anti-IL-1 1 treatment synergized to reverse impaired Tfh cell and virus-specific antibody production in hDPP4-Pla2g2d-/- mice. (A) Protocol for rDC adoptive transfer and IL-1 $\beta$ blockade in infected hDPP4-Pla2g2d-/- mice. (B) Serum MERS-CoVspecific PRNT $_{50}$ in mice were determined at indicated days after immunization with sublethal dose of MERS-CoV, $n=4 /$ group. (C and D) Immunized mice were challenged with a lethal dose of MERS-CoV and monitored for survival (C) and virus load (D), $n=5$ to 6/group. (E and F) Serum MERS-CoV-specific PRNT $_{50}(\mathbf{E})$ and Tfh cell numbers $(\mathbf{F})$ in lungs and spleen were determined at indicated days after challenge, $n=4 /$ group. (B-F) Data are shown as mean \pm SEM and are representative of 3 independent experiments. Data shown in $\mathbf{B}, \mathbf{E}$, and $\mathbf{F}$ were compared using multiple regression analysis, while data shown in $\mathbf{D}$ were analyzed using Student's $t$ test. ${ }^{*} P<0.05,{ }^{* *} P<0.01$.

tracking of the virus-specific Tfh response after immunization. After i.n. immunization with VRP-MERS-N, N99-specific CD4+ T cells were detected at equivalent levels in the spleens and lungs of middle-aged $h D P P 4$ and $h D P P 4-P l a 2 g 2 d^{-/}$mice (Figure 4, D and E). However, virtually no N99-specific CXCR $5^{+} \mathrm{PD}-1^{+} \mathrm{CD} 4^{+} \mathrm{T}$ cells were detected in hDPP4-Pla2g2d $d^{-/}$lungs or spleens (Figure 4E). Consistent with these data, Bcl-6 was decreased in N99-specific hDPP4-Pla2g2d $d^{--} \mathrm{CD} 4^{+} \mathrm{T}$ cells (Figure $4 \mathrm{~F}$ ). Follicular regulatory $\mathrm{T}$ cells (Tfr) expressing Foxp3 inhibit Tfh cell function (28-31). However, frequencies of Foxp3 $3^{+} \mathrm{N} 99^{+} \mathrm{CD} 4^{+} \mathrm{Tfr}$ were very low but similar in both $h D P P 4$ and $h D P P 4-P l a 2 g 2 d^{--}$mice (Figure $4 \mathrm{~F}$ and Supplemental Figure 5D), making it unlikely that these cells contribute to the deficiency in Tfh cells in middle-aged hDPP4Pla2g2 $d^{-/}$mice. Moreover, transcription factors associated with other Th subsets were comparable between hDPP4 and hDPP4Pla2g2 $d^{-/}$mice (Supplemental Figure 5D).

After N99 peptide stimulation, equivalent numbers of cells expressed IFN- $\gamma$ in the lungs and spleens, but IL-21-expressing cells were largely absent in $h D P P 4-P l a 2 g 2 d^{-/}$mice. IL-2, an inhibi- tor of Tfh cell development (32), and TNF were expressed at higher levels in these mice (Figure 4G). Therefore, PLA G2D deficiency changed the profile of cytokine expression by $\mathrm{CD} 4^{+} \mathrm{T}$ cells and resulted in a dramatic impairment of Th cell formation.

Impaired Tfh cell formation in middle-aged hDPP4-Pla2g2 $d^{-1}$ mice is not $T$ cell intrinsic. To determine whether the absence of $\mathrm{Tfh}$ cell formation after i.n. immunization is $\mathrm{T}$ cell intrinsic, we cotransferred splenic CD4 ${ }^{+} \mathrm{T}$ cells from 6-month-old Thy1 and CD45-mismatched unimmunized mice ( Pla2g $2 d^{++}{ }^{+} C D 45.1^{+}$Thy $1.2^{+}$ and Pla2g2d ${ }^{-{ }^{-} C D} 45.2^{+}$Thy $1.2^{+}$) into Pla2g2d $d^{++} C D 45.2^{+}$Thy $1.1^{+}$ mice prior to immunization with a single sublethal dose of MERS$\mathrm{CoV}$ (Supplemental Figure 6A). Sublethal MERS-CoV immunization was used in these experiments because VRP-MERS-S/N vaccination is a 2-step process, requiring priming and boosting. On 10,20 , and 30 days after immunization, lungs, DLNs, and spleens of recipients were analyzed. CD $4^{+} \mathrm{T}$ cells from Pla2g2d ${ }^{+/+}$and Pla2g2 $\mathrm{d}^{-/}$mice differentiated equivalently into Tfh cells, suggesting a T cell-extrinsic defect in Pla2g2 $\mathrm{d}^{-/}$mice (Supplemental Figure 6, B and C). Further, Tfh cell survival was not grossly impaired 
A
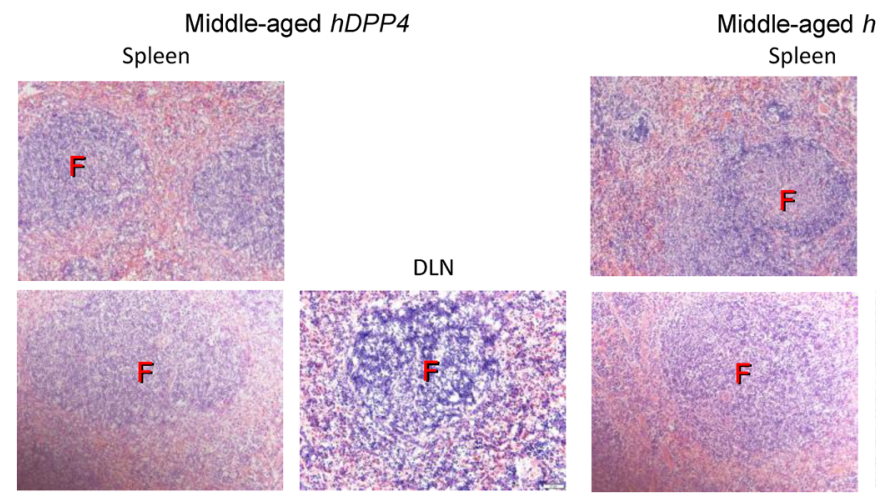

Naive
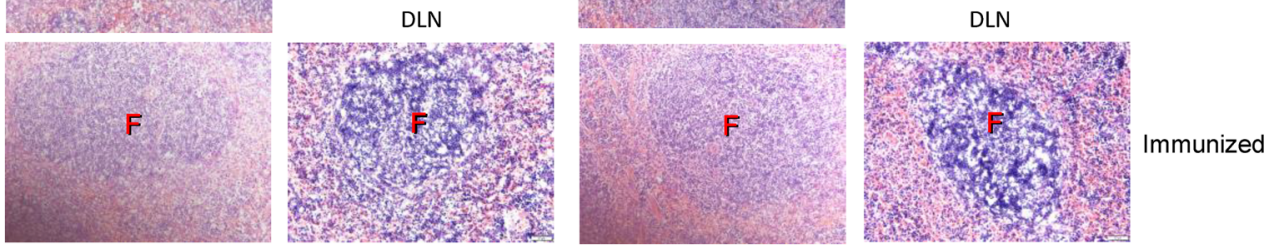

B

Immunized and infected middle-aged mice
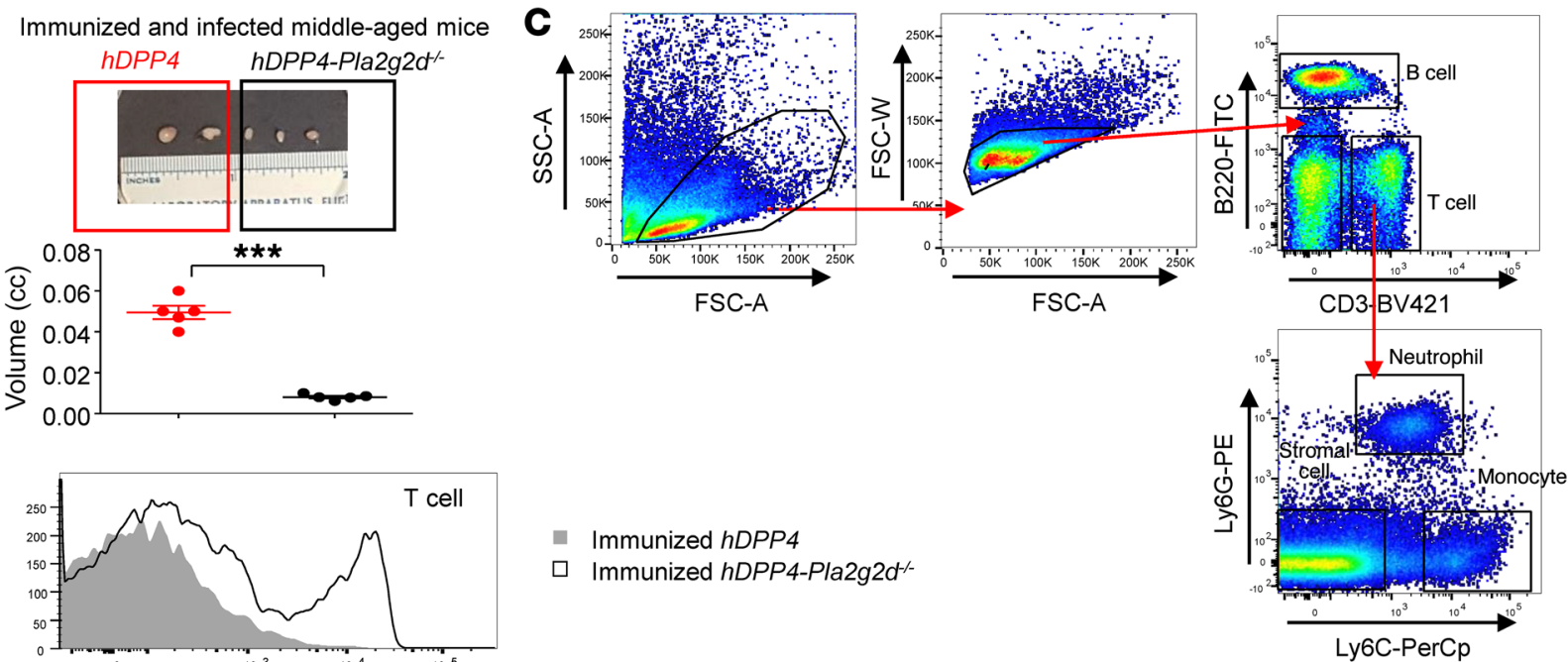

D

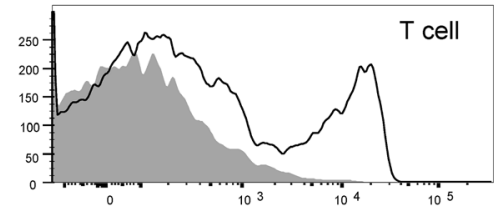

- Immunized hDPP4

$\square$ Immunized hDPP4-Pla2g2d ${ }^{-1-}$
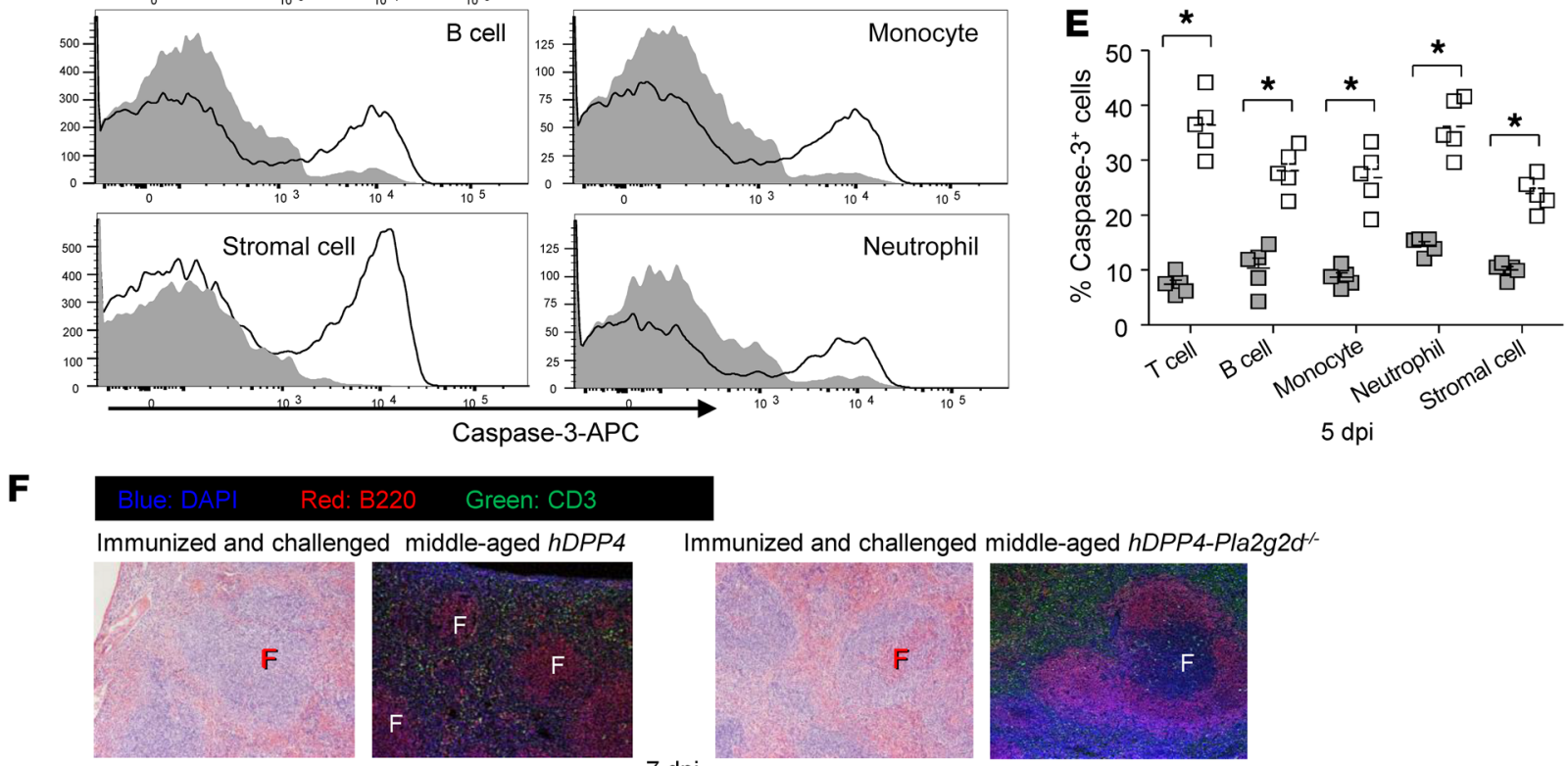

Green: CD3
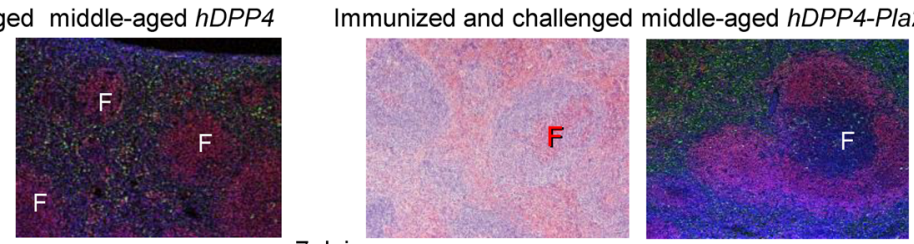

$7 \mathrm{dpi}$

Figure 7. Increased DLN cell apoptosis and aberrant spleen follicle formation in infected hDPP4-PIa2g2d-/- mice. (A) Five-month-old $h D P P 4$ and $h D P P 4-$ Pla2g2d $d^{-1-}$ mice were immunized with VRP-MERS-S or PBS as described in Figure 1D and challenged i.n. with a lethal dose of MERS-CoV. H\&E staining of spleens and DLNs from naive and immunized middle-aged $h D P P 4$ or $h D P P 4-P l a 2 g 2 d^{-1-}$ mice (sacrificed on day 0 or day 70 after immunization with VRP-MERS-S) is shown. Original magnification $\times 400$. (B) DLN from middle-aged $h D P P 4$ or $h D P P 4-P l a 2 g 2 d^{-/-}$mice were harvested on $7 \mathrm{dpi}$. Note small size

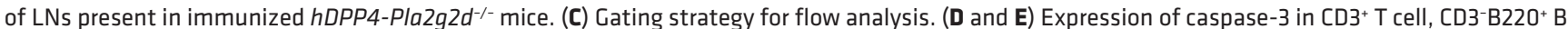

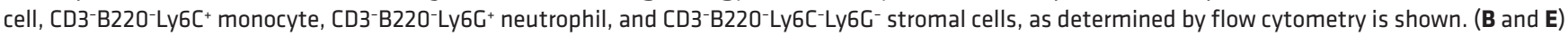
Summary data are shown and are representative of 3 independent experiments. Data are shown as mean \pm SEM and analyzed using Student's $t$ test, $n=$ 5/group. ${ }^{*} P<0.05,{ }^{* *} P<0.001$. (F) Confocal image of $h D P P 4$ and $h D P P 4-P l a 2 g 2 d^{-/-}$spleens harvested on $7 \mathrm{dpi}$. F, follicle. 
in Pla2g $2 d^{-/-}$mice because Tfh cells transferred from $h D P P 4$ mice partially reversed the defect in MERS-CoV-neutralizing antibody production observed in the absence of PLA2G2D expression (Supplemental Figure 6D).

Impaired antibody production and Th cell formation in middle-aged hDPP4-Pla2g2d $d^{-/}$mice is reversed by adoptive transfer of $W T C D 11 c^{+}$rDCs and $I L-1 \beta$ blocking. Together, the data shown above indicated that deficient antibody generation in $\mathrm{Pla} 2 \mathrm{~g} 2 \mathrm{~d}^{-/}$ mice was not $\mathrm{T}$ cell intrinsic. Since DCs are required for the initial steps in Tfh cell differentiation (23) and PLA ${ }_{2}$ G2D is expressed primarily by DCs (12), we next assessed whether DC dysfunction contributed to the impaired development of Tfh cells in Pla2g2 $\mathrm{d}^{-/}$ mice. Of note, when spleen DCs and rDCs (CD3-CD19-CD56 MHC-II' ${ }^{+}$CD64-, gating shown in Supplemental Figure 7A) from immunized $h D P P 4$ mice were analyzed, rDCs but not spleen DCs expressed higher levels of Pla2g2d (Supplemental Figure 7B). Furthermore, we found that the absolute number of DCs was higher in DLNs and spleens of hDPP4-Pla2g2 $d^{-/-}$compared with $h D P P 4$ mice after immunization with a sublethal dose of MERS-CoV, although only the DLN differences were statistically significant (Figure 5A). To determine whether these differences in DC numbers reflected rDC trafficking from the lungs, rDCs were labeled by i.n. administration of carboxyfluorescein succinimidyl ester (CFSE) 18 hours prior to DLN harvest. There was a dramatic increase in the numbers of lung-derived $\mathrm{CFSE}^{+}$DCs in the DLNs, measured at day 3 postinfection ( $3 \mathrm{dpi}$ ) (Figure $5 \mathrm{~A}$ ).

Since $\mathrm{CD} 11 \mathrm{c}^{+} \mathrm{rDCs}$ were more activated during acute respiratory virus infection in Pla2g2 $\mathrm{d}^{-/}$compared with control mice, we next assessed their activation status after immunization by analyzing proinflammatory cytokine expression. A greater proportion of hDPP4-Pla2g2d $d^{-/-}$compared with $h D P P 4$ rDCs expressed pro- and cleaved IL-1 3 (Figure 5B and Supplemental Figure 7C). No differences were detected in expression of MHC-I, MHC-II, CD80, CD83, and CD86 by rDCs harvested from the 2 strains of mice (Figure $5 \mathrm{~B}$ ). This correlation between decreased $\mathrm{Tfh}$ cell production and elevated IL- $1 \beta$ expression was unexpected since IL-1 $\beta$ is required for Tfh cell generation $(33,34)$. To examine the possibility that greater than normal levels of IL-1 $\beta$ actually diminish Tfh cell formation, we treated CD11 $\mathrm{c}^{+}$rDCs and T cell cocultures in vitro under conditions that allowed the outgrowth of Tfh cells. Increased amounts of IL-1 $\beta$ resulted in an increase in the proportion of Tfh cells in the culture, but this enhancement was reversed when IL-1 $\beta$ amounts were further increased (Figure 5C). Similar effects were not observed on the expansion of IL-2- or IL-17expressing $\mathrm{CD}^{+} \mathrm{T}$ cells (Figure $5 \mathrm{C}$ ).

Since these results suggest that $\mathrm{CD} 11 \mathrm{c}^{+} \mathrm{rDC}$ function is modulated by the absence of PLA ${ }_{2}$ G2D, we next examined whether CD $11 \mathrm{c}^{+} \mathrm{rDCs}$ from WT mice could correct the antibody and Tfh cell defects observed in hDPP4-Pla2g $2 d^{-/}$mice. We transferred purified $C D 11 c^{+}$rDCs from $h D P P 4$ and $h D P P 4-P l a 2 g 2 d^{-/}$mice i.n. into recipient $h D P P 4-P l a 2 g 2 d^{-/-}$mice on days 0 and 3 after sublethal MERS-CoV infection. CellTrace labelling showed that transferred hDPP4 and hDPP4-Pla2g2d $d^{-/}$rDCs migrated into recipient DLNs with similar efficiency (Supplemental Figure 7D), and that transferred $\mathrm{rDCs}$ represented the major source of IL-1 $\beta$ in DLNs (Supplemental Figure 7E). However, rDC transfer alone did not correct the defect in antibody and Tfh cell development in hDPP4-Pla2g2 $d^{-/-}$mice (Figure 5D). Since IL-1 $\beta$ was the only cytokine that differed in expression by CD11 $\mathrm{c}^{+}$rDCs (Figure 5B) and increased levels of IL- $1 \beta$ resulted in decreased Tfh cell generation (Figure $5 \mathrm{C}$ ), we next treated mice with low levels of IL-1 $\beta$ blocking antibody (Supplemental Figure 8A), to minimize the effects of IL-1 $\beta$ expressed by recipient hDPP4-Pla $2 g 2 d^{-/-} \mathrm{rDCs}$, while not affecting Tfh cell differentiation (33-36). Unlike higher doses of anti-IL-1 $\beta$ antibody, this dose $(1 \mu \mathrm{g} / \mathrm{g}$ weight $)$ did not delay virus clearance or inhibit $\mathrm{CoV}$-specific antibody production (Supplemental Figure 8B). We then challenged mice at $28 \mathrm{dpi}$. By itself, IL-1 $\beta$ blockade failed to reverse decreased survival or impaired neutralizing antibody production (Supplemental Figure $8 \mathrm{C}$ ). However, transfer of $\mathrm{CD}_{11 \mathrm{c}^{+}} \mathrm{rDC}$ from $h D P P 4$ mice in conjunction with anti-IL-1 $\beta$ antibody (Figure 6A) resulted in greater survival compared with those receiving $\mathrm{CD} 11 \mathrm{c}^{+} \mathrm{rDCs}$ from $h D P P 4-P l a 2 g 2 d^{-/}$mice (Figure 6C). More impressively, defects in kinetics of virus clearance after challenge (Figure 6D), neutralizing antibody production (Figure 6, B and E), and Tfh cell numbers in the lung (Figure 6F) were reversed. Taken together, these results suggest that $\mathrm{PLA}_{2} \mathrm{G} 2 \mathrm{D}$ expression specifically in lung CD11 $\mathrm{c}^{+}$rDCs is critical for the development of Tfh cells and production of virus-specific antibody.

Increased DLN cell apoptosis and aberrant splenic follicle architecture in immunized hDPP4-Pla2g2d $d^{-/}$mice after virus challenge. To begin to understand the basis of this rDC-specific defect, we analyzed the architecture of the DLN and spleen, since these are primary sites for initiation and activation of adaptive immune responses after lung infection. We observed no differences in the structure of the spleen or DLN when naive and immunized (Figure 7A) middle-aged $h D P P 4$ and hDPP4-Pla2g2d $d^{-/-}$mice were compared. However, we reasoned that effects might be more obvious after immunization and subsequent challenge, so we similarly analyzed lymphoid tissue in MERS-CoV-infected middle-aged hDPP4 and hDPP4-Pla2g2 $d^{-/}$mice that had been previously immunized. At 7 dpi, DLN size was greatly decreased in hDPP4Pla2g2 $d^{-/}$compared with $h D P P 4$ mice (Figure $7 \mathrm{~B}$ ), concomitant with increased lymphocyte, myeloid cell, and stromal cell apoptosis (Figure 7, C-E). Further, splenic architecture was grossly abnormal at 7 dpi and was characterized by infiltration of CD3 $\mathrm{T}$ cells into the follicles of infected $h D P P 4-P L A_{2} G 2 D^{-/-}$spleens (Figure 7F). Taken together, these results suggest that enhanced DC migration from lungs and enhanced rDC activation resulted in excessive inflammation in lymphoid organs of immunized and challenged $h D P P 4-P l a 2 g 2 d^{-/-}$mice, leading to cellular apoptosis. These effects were greatest on Tfh cells with consequent effects on virus-specific antibody production.

$P L A_{2} G 2 D$ deficiency modifies the inflammatory status of human monocyte-derived macrophages (MDMs) and DCs (MDDCs). Finally, to determine the potential clinical relevance of PLA ${ }_{2}$ G2D in modulating DC and macrophage function, we inactivated PLA2G2D gene expression in primary PBMC-derived human MDDCs using CRISPR-Cas9 editing (Supplemental Figure 9, A and B). In vitro generation of human Tfh cells has not yet been described, so we could not directly assess the effect of PLA2G2D deletion on Tfh cell production. As a surrogate approach, we infected control and PLA2G2D ${ }^{-/}$human MDDCs with MERS-CoV or treated them with poly I-C, a TLR3 agonist. We detected greater expression of IL-1 $\beta$ 
in $P L A 2 G 2 D^{-/-}$compared with PLA2G2D $D^{+/+}$MDMs and MDDCs (Supplemental Figure 9C), consistent with results obtained using mouse $P L A 2 G 2 D^{-/-}$rDCs (Figure $5 \mathrm{~B}$ ).

\section{Discussion}

Here, we show that PLA $\mathrm{P}_{2} \mathrm{D}$ is critical for the development of antibody responses against several coronaviruses, including SARS-CoV, MERS-CoV, and SARS-CoV-2. The effects are confined to i.n. immunization and occur in middle-aged mice. We showed previously that age-dependent increased expression of Pla2g2d in rDCs played a role in increased susceptibility to these viruses during acute infection and that genetic deletion of Pla2g2d resulted in enhanced $\mathrm{T}$ cell responses with concomitant enhanced kinetics of virus clearance. We postulated that Pla2g2d was increased in response to low level increases in inflammation that occur with aging, as a counter measure to protect the host from tissue damage. Consistent with this, interventions that decreased oxidative stress in middle-aged mice resulted in diminished expression of Pla2g2d mRNA (13). However, our results show that increased Pla2g2d expression has an unexpected role in facilitating the development of virus-specific antibody responses in the lung during aging. This enhancing effect, like its deleterious effect in acute infection, is a consequence of its antiinflammatory properties. We note that SARS, MERS, and COVID-19 are most severe in the elderly, so the effects of $\mathrm{PLA}_{2} \mathrm{G} 2 \mathrm{D}$ in mice are apparent at a younger age than severe disease occurs in most patients.

We showed a lack of protection after i.n. immunization of Pla2g2 $d^{-/}$but not Pla2g2 ${ }^{+/+}$mice with sublethal amounts of MERS-CoV or SARS-CoV-2 infection or with a VRP-based vaccine and challenge with high doses of the cognate viruses. Immunization was not protective because $\mathrm{Pla} 2 \mathrm{~g} 2 \mathrm{~d}^{-/}$mice failed to generate virus-specific antibody responses (Figure 2, Supplemental Figure 2). As in mice with acute respiratory viral disease, the absence of PLA $\mathrm{A}_{2} \mathrm{D}$ resulted in greater proinflammatory responses, including expression of proinflammatory cytokines, but in these immunized mice, the effects were detrimental. These augmented immune responses were most apparent after immunization and challenge. They resulted in increased myeloid cell apoptosis (Figure 7, C-E), an absence of germinal center formation, and profound defects in Th cell differentiation with subsequent impairment of the anti-virus antibody response. This defect was not found in young Pla2g2 ${ }^{-/}$mice (Figure 2, B and D), suggesting an indispensable role for age-dependent increases in PLA ${ }_{2}$ GD expression in the lungs. Notably, while we think that the same processes contributed to a lack of an antibody response after i.n. immunization in the absence of challenge, we could not demonstrate any changes in myeloid cell apoptosis or in germinal center formation after immunization alone (Figure 7A), even though Tfh cell differentiation was still impaired (Figure 4, A and B). These results indicate that gross disruption of germinal centers was not required for deficient immune memory formation.

A key finding was that the $\mathrm{CD} 4^{+} \mathrm{Tfh}$ cell lineage was preferentially affected by the absence of PLA ${ }_{2}$ GD. A notable difference between Pla2g $2 \mathrm{~d}^{-/}$and Pla2g $2 \mathrm{~d}^{+/+}$rDCs was enhanced IL-1 $\beta$ expression by mouse Pla2g2 $\mathrm{d}^{-/-}$and human PLA2G2D $\mathrm{D}^{-/-} \mathrm{DCs}$, which has been found to contribute to an increased inflammatory milieu in DLNs (Figure 5B, Figure 7, and Supplemental Figure
9C) $(37,38)$. A downstream product of $\mathrm{PLA}_{2} \mathrm{G} 2 \mathrm{D}$ is prostaglandin D2 $\left(\mathrm{PGD}_{2}\right)$, which, on signaling through its receptor on myeloid cells (DP1), inhibits inflammasome activation and IL-1 $\beta$ expression (13), thus providing a link between the absence of PLA 2 G2D and augmented IL-1 $\beta$ expression. Partial IL-1 $\beta$ blockade by itself did not correct the deficiency in Tfh cell numbers or anti-MERS$\mathrm{CoV}$ antibody production in Pla2g2 $\mathrm{d}^{-/-}$mice (Supplemental Figure $8 \mathrm{C})$, suggesting that additional factors provided by cotransferred WT CD11 ${ }^{+}$rDC were required to correct the observed deficiency (Figure 6). Additional work will be required to identify these factors and to understand more precisely how Pla2g2 $\mathrm{d}^{-/-}$DCs and IL-1 $\beta$ contribute to suboptimal virus-specific antibody and Tfh cell responses after immunization alone.

Increased IL-2 expression by T cells (Supplemental Figure 1, $\mathrm{C}$ and D) could also contribute to poor Tfh cell development in infected Pla2g $2 d^{-/}$mice. IL-2 enhances conventional T cell and suppresses Tfh cell differentiation (32), and also contributes to the conversion of Th to non-Tfh cells, resulting in loss of B cells and immunoglobulin production (39). In addition, TNF production by activated $\mathrm{T}$ cells was increased in the absence of PLA $\mathrm{F}_{2} \mathrm{D}$ (Supplemental Figure 1, C and D) and TNF has been shown to augment pro-IL-1 $\beta$ synthesis in myeloid cells (40). This positive feedback may exacerbate the proinflammatory milieu in lymphoid organs. Of note, preferential loss of Tfh cells has also been observed in patients with COVID-19 and was attributed to an increase in T-bet ${ }^{+}$ Th1 cells and aberrant extrafollicular TNF accumulation (41).

Collectively, these results suggest that elevated IL-1 $\beta$ expression by $\mathrm{CD} 11 \mathrm{c}^{+}$rDCs in conjunction with altered expression of other proinflammatory cytokines such as IL-2 and TNF contributes to a lack of Tfh cell differentiation and to enhanced lymph node cell apoptosis in Pla2g2 $d^{-/-}$mice. PLA ${ }_{2}$ G2D in the lungs may serve as an immune rheostat, controlling rDC activation and expression of molecules such as CCR7 (42), important for migration to DLN and T cell priming, and thereby minimizing immune recognition of innocuous antigens. However, in the absence of this antiinflammatory molecule, rDCs appear to be more activated during aging, with effects on myeloid cell and lymphocyte survival and Th cell development and antibody responses after vaccination and respiratory virus infection. Since aging is a risk factor for severe coronavirus disease and mortality (2-4) and inflammaging occurs in human lungs, these results raise the possibility that $\mathrm{PLA}_{2} \mathrm{G} 2 \mathrm{D}$ has a critical role in optimizing immune responses, including the antibody response in the elderly infected with any of these coronaviruses.

Several limitations to this study should be noted. Only mice raised in an SPF (specific pathogen-free) environment were used. Extension of the studies to non-SPF young and aged mice might be more relevant for understanding the role of PLA 2 G2D in humans. Another limitation is the lack of confirmation using human respiratory tract samples. Comparisons among samples obtained from young and aged healthy and SARS-CoV-2-infected mice will be important for translating the results to human populations.

\section{Methods}

Mice, virus, and infection. Specific pathogen-free 8-week-old to 6-month-old C57BL/6 mice (B6, CD45.2, or CD45.1) were purchased from Charles River Laboratories. Thy1.1 (B6) mice were purchased 
from Jackson Laboratories. $h D P P 4-K I$ mice were generated and maintained at the University of Iowa (16). hDPP4-KI mice and Pla2g2d -/ mice (12) were crossed to generate hDPP4-Pla2g2 $d^{-/-}$mice. All data shown in this manuscript were obtained from male mice.

MERS-CoV (EMC2012 strain, passage 8) was provided by Bart Haagmans and Ron Fouchier (Erasmus Medical Center, Rotterdam, Netherlands). SARS-CoV and MERS-CoV were propagated on Vero E6 and Vero81 cells, respectively. Vero cells were grown in DMEM (GIBCO) supplemented with 10\% FBS. The 2019n-CoV/USA-WA1/2019 strain of SARS-CoV-2 (accession no. MT985325.1) used in these studies was obtained from the CDC and the mouse-adapted SARS-CoV-2 strain was generated using a bacterial artificial chromosome-based lambda red recombination system as previously described (43). SARSCoV-2 virus was passaged on Calu-3 2B4 cells. Calu-3 2B4 cells were grown in MEM (GIBCO) supplemented with $20 \%$ FBS.

For infections, mice were lightly anesthetized using isoflurane and i.n. infected with lethal dose (250 pfu for young mice or $750 \mathrm{pfu}$ for middle-aged mice) MERS-CoV or $1 \times 10^{4}$ pfu SARS-CoV in a 50 $\mu \mathrm{L}$ volume respectively. For SARS-CoV-2 infection, 6-month-old C57BL/6 mice were transduced with $2.5 \times 10^{8}$ pfu Ad5-hACE2. Five days later, transduced mice were infected with $1 \times 10^{5} \mathrm{pfu}$ SARS-CoV-2 in a $50 \mu \mathrm{L}$ volume $\operatorname{DMEM}(18,44)$.

Immunization protocol. For VRP immunization, mice were lightly anesthetized using isoflurane and inoculated with $2 \times 10^{6}$ pfu VRPMERS-S, VRP-MERS-N, or VRP-SARS-S i.n. in $50 \mu \mathrm{L}$ respectively. Four weeks later, mice were boosted with the same dose of VRPs. For i.p. immunization, mice were immunized and boosted with $2 \times 10^{6} \mathrm{pfu}$ VRPMERS-S in $200 \mu \mathrm{L}$. For immunization with infectious virus, mice were lightly anesthetized using isoflurane and infected with a sublethal dose of MERS-CoV (100 pfu), or SARS-CoV-2 $\left(10^{5} \mathrm{pfu}\right)$, i.n. in a $50 \mu \mathrm{L}$ volume.

Virus titration. Lungs were harvested on the indicated days after infection. Tissues were homogenized in PBS using a manual homogenizer and titered on Vero E6 (for SARS-CoV and SARS-CoV-2) or Vero81 cells (for MERS-CoV) respectively. For plaque assays, cells were fixed with $10 \%$ formaldehyde 3 days later and stained with crystal violet.

Plaque reduction neutralization test ( $\left.P R N T_{50}\right)$. Serum or antibody samples were serially diluted in DMEM and mixed 1:1 with 80 pfu MERS$\mathrm{CoV}$, SARS-CoV, or SARS-CoV-2 for 1 hour at $37^{\circ} \mathrm{C}$. The mixtures were then added into Vero81 cells (for MERS-CoV) or Vero E6 (for SARS-CoV and SARS-CoV-2) for an additional 1 hour at $37^{\circ} \mathrm{C}$. After removing the culture medium, cells were overlaid with $1.2 \%$ agarose and cultured for 3 days. Plaques were visualized by $0.1 \%$ crystal violet staining. PRNT $_{50}$ was determined as the concentration of serum required to reduce the number of plaques by $50 \%$ compared with control serum-exposed virus.

Histology and immunohistochemistry. Animals were anesthetized and transcardially perfused with PBS followed by zinc formalin. Lungs, DLNs, and spleens were removed, fixed in zinc formalin, and paraffin embedded. Sections were stained with hematoxylin and eosin and examined by light microscopy. Images were acquired using a BX61 light microscope (Olympus) and CellSens software (Olympus). Sections were evaluated using postexamination masking techniques for ordinal scoring (45). To detect hACE2 expression in lungs, sections were incubated with blocking reagent (Rodent Block-M, Biocare Medical) incubated with a mouse monoclonal antibody to hACE2 (1:100 dilution, mouse anti-hACE2, MAB933, R\&D Systems), then incubated with a secondary (polymer-based) kit (Mouse Envision, Dako), followed by incubation with DAB+ (Dako), then incubated with Rabbit Envision (Dako) and diaminobenzidine (Dako).
Confocal microscopy. Tissues were harvested as described above and fixed with $4 \%$ PFA at $4{ }^{\circ} \mathrm{C}$ for 4 hours, followed by immersion in $10 \%, 20 \%$, and $30 \%$ sucrose in PBS for 12 hours each. Sections 5 to 15 $\mu \mathrm{m}$ thick were then prepared from OCT-embedded samples and fixed in acetone for 10 minutes at $4^{\circ} \mathrm{C}$. For staining, sections were blocked with goat serum for 2 hours in humidity chambers at room temperature (RT). Sections were then treated with primary antibodies (rabbit anti-mouse CD3, SP7; rat anti-mouse B220, MAB1217; R\&D Systems) at $4^{\circ} \mathrm{C}$ overnight. After washing with $\mathrm{PBS}$, samples were treated with secondary antibodies (Alexa 647-goat anti-rabbit IgG, Alexa 568-goat anti-rat IgG, Abcam) for 30 minutes at RT. Finally, slides were overlaid with antifade mounting medium containing DAPI (Vector) and examined using a confocal microscope (Zeiss 710).

Antibodies and flow cytometry. The following monoclonal antibodies were used: APC-eFluor780-conjugated mouse anti-human CD8 (clone SK1), PE-conjugated mouse anti-human TNF (clone MAB11), PE or PercP Cy5.5-conjugated rat anti-mouse CD4 (clone RM4-5), FITC-conjugated rat anti-mouse CD8 (clone 53-6.7), PercP Cy5.5-conjugated mouse anti-mouse Ly6C (clone HK1.4), APC-conjugated rat anti-mouse F4/80 (clone BM8), PE-conjugated hamster anti-mouse CD103 (clone 2E7), PE-conjugated mouse anti-mouse GATA3 (clone TWAJ), PE-conjugated mouse anti-mouse ROR $\gamma \mathrm{t}$ (clone B2D), PercP Cy5.5 or eFluor-conjugated mouse anti-mouse Foxp3 (clone FJK-16s), APC-conjugated rat anti-mouse IL-1 $\beta$ (clone NJTEN3), PE-conjugated mouse anti-mouse IL-21 (clone mhalx21) and rat anti-mouse CD16/32 (clone 2.4G2) (eBioscience); PerCP Cy5.5 or Brilliant Violet (BV) 510-conjugated mouse anti-human CD4 (clone RPA-T4), PerCP Cy5.5-conjugated hamster anti-mouse CD3 (clone 145-2C11), BV421-conjugated mouse anti-mouse CD45.1 (clone A20), APC-conjugated mouse anti-mouse Thy1.1 (clone OX-7), PE-Cy7-conjugated mouse anti-mouse NK1.1 (clone PK136), PerCP Cy5.5 or PE-conjugated rat anti-mouse CD45 (clone 30-F11), PerCP Cy5.5-conjugated mouse anti-mouse CD38 (clone 90), PE-conjugated-anti-mouse CD64 (clone X54-5/7.1), FITC-conjugated mouse anti-mouse CD138 (clone DL-101), PE-Cy7-conjugated mouse anti-mouse MHC-I (clone 28-8-6), BV510-conjugated mouse anti-mouse MHC-II (clone M5/114.15.2), PE-conjugated mouse antimouse CD80 (clone 2D10), PE-Cy7-conjugated mouse anti-mouse CD83 (clone HB15e), APC-Vy7- or BV510-conjugated rat antimouse CD11b (clone M1/70), APC-conjugated hamster anti-mouse CD11b (clone N418), PE-Cy7-conjugated mouse anti-mouse PD-1 (clone RPM1-30), FITC-conjugated mouse anti-mouse XCR1 (clone ZET), APC/Fire 750-conjugated goat anti-rat IgG (clone poly4054), APC-conjugated mouse anti-human IFN- $\gamma$ (clone B27), PE-conjugated mouse anti-human perforin (clone B-D48), APC-conjugated rat anti-mouse perforin (clone S16009B), FITC-conjugated mouse anti-human/mouse Granzyme B (clone QA16A02), BV421-conjugated mouse anti-mouse T-bet (clone 4B10), PerCP Cy5.5-conjugated mouse anti-mouse Bcl-6 (clone 7D1), Alexa Fluor 488-conjugated rat anti-mouse IL-2 (clone JES6-5H4), APC-conjugated rat anti-mouse IL-6 (clone MP5-20F3), Alexa Fluor 647-conjugated rat anti-mouse IL-10 (clone JES5-16E3), PE-conjugated rat anti-mouse IL-12 (clone C15.6), Alexa Fluor 488, APC-Cy7, or APC-conjugated rat anti-mouse IFN- $\gamma$ (clone XMG1.2), and APC-conjugated rat antimouse TNF (clone MP6-XT22, BioLegend). BV421-conjugated hamster anti-mouse CD3 (clone 145-2C11), FITC-conjugated mouse anti mouse B220 (clone RA3-6B2), APC-conjugated rat anti-mouse Ly6G 
(clone 1A8), FITC-conjugated rat anti-mouse CD86 (clone GL1), rabbit anti-mouse cleaved caspase 3 (Abcam); FITC or BV421-conjugated hamster anti-mouse CD11c (clone HL3), rat anti-mouse CXCR5 (clone 2G8, BD Biosciences). For surface staining, $1 \times 10^{6}$ cells were blocked with $1 \mu \mathrm{g}$ anti-CD16/32 antibody (BD Biosciences) and stained with the indicated antibodies at $4^{\circ} \mathrm{C}$. For CXCR5 staining, cells were stained with unconjugated anti-CXCR5 antibody at $37^{\circ} \mathrm{C}$ for 1 hour followed by secondary antibody at RT for 30 minutes. For intracellular molecule and cytokine staining, cells were fixed and permeabilized using Cytofix/Cytoperm Solution (BD Biosciences) after washing and stained with the indicated antibodies. To detect antigen-specific T cells after MERS-CoV infection, $1 \times 10^{6}$ cells were cultured in 96-well round bottom plates in the presence of Brefeldin $\mathrm{A}$ (BFA) and stimulated with pools of $\mathrm{N}$ or $\mathrm{S}$ peptide pools or a $\mathrm{CD} 4^{+}$ T cell epitope peptide (N99, YFYYTGTGPEAALPF) (Bio-Synthesis). N99-specific CD4 ${ }^{+} \mathrm{T}$ cells were also identified using APC-conjugated I-A $/$ N99 tetramers (NIH Tetramer Facility) after staining at $37^{\circ} \mathrm{C}$ for 6 hours. To determine the absolute number of cells, CountBright absolute counting beads (Invitrogen) were added during staining. A LIVE/DEAD cell viability assay kit (Invitrogen) was used for gating live cells and labeling target cells in cytotoxicity assays. Flow cytometric data were acquired using a FACSVerse and were analyzed using FlowJo software (Tree Star).

ELISA assay for total immunoglobulins. Total immunoglobulins in sera and BAL were measured by ELISA and quantitated using a mouse IgG standard (Thermo Fisher Scientific). Briefly, plates were coated with serially diluted samples at $4^{\circ} \mathrm{C}$ for 4 hours. HRP-conjugated goat anti-mouse IgG, IgA, IgM and IgE antibodies (Thermo Fisher Scientific) were added and visualized by addition of substrate. OD450 values were read with a Synergy hybrid reader H1 (BioTek).

ELISPOT assay. ELISPOT plates (Millipore) were coated with lysates from VRP-MERS-S- or VRP-MERS-N-transduced cells at $4^{\circ} \mathrm{C}$ for 4 hours. After washing, splenocytes isolated from immunized middle-aged hDPP4 or hDPP4-Pla2g2d-/ mice were seeded onto the coated plates at $10^{6} \mathrm{cells} /$ well in triplicate and cultured at $37^{\circ} \mathrm{C}$ overnight. Antibody-secreting $\mathrm{B}$ cells were then detected with HRP-conjugated antimouse antibodies, visualized by addition of substrate and counted.

Adoptive transfer of $\mathrm{T}$ lymphocytes. $\mathrm{CD} 4^{+} \mathrm{T}$ cells isolated from mid-

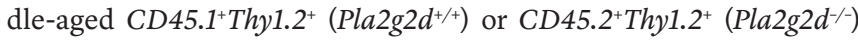
mouse spleens and purified with a mouse $\mathrm{CD} 4^{+} \mathrm{T}$ cell isolation kit II (Miltenyi Biotec) were mixed at a ratio of 1:1 and adoptively transferred intravenously into CD $45.2^{+}$Thy $1.1^{+}\left(\right.$Pla2g $\left.2 d^{+/+}\right)$mice at $10^{7}$ cells/ mouse. One day after transfer, mice were infected i.n. with a sublethal dose of MERS-CoV. Lungs, DLNs, and spleens were harvested at the indicated time points and analyzed for host and donor Tfh cells.

CD11 $c^{+}$rDCs magnetic bead separation. Lung and DLN single-cell suspensions were prepared. CD11 $\mathrm{c}^{+}$DCs were purified by a 2-step process. Initially cells were negatively selected using a pan mouse DC isolation kit (Miltenyi Biotec). Cells were then exposed to anti-mouse CD11c MicroBeads (Miltenyi Biotec) and isolated using an autoMACS system (Miltenyi Biotec) following the manufacturer's protocol.

Adoptive transfer of CD11c rDCs. A quantity of $10^{6} \mathrm{CD}^{+} 11 \mathrm{c}^{+} \mathrm{rDCs}$ isolated from lung and lung-DLNs of middle-aged hDPP4 or hDPP4$P l a 2 g 2 d^{-1-}$ mice were adoptively transferred by i.n. inoculation into hDPP4-Pla2g2 $\mathrm{d}^{-/-}$mice at days 0 and 3 after immunization with a sublethal dose of MERS-CoV. In some experiments, cells were stained with CellTrace Violet Cell Proliferation Kit (Invitrogen) prior to transfer.
In vitro differentiation of Tfh cells. Mouse $\mathrm{CD} 4^{+} \mathrm{T}$ cells were purified from splenocytes of WT mice by microbeads and seeded onto 96-well flat-bottom plates $\left(5 \times 10^{5}\right.$ cells/well). CD11 $\mathrm{c}^{+} \mathrm{rDCs}$ were then purified from lungs and DLNs and added into plates at a T/DC ratio of $4: 1$ in the presence of $1 \mu \mathrm{g} / \mathrm{mL}$ MERS-CoV-S peptide, $100 \mathrm{ng} / \mathrm{mL}$ IL-6, and $50 \mathrm{ng} / \mathrm{mL}$ IL-21 (PeproTech), and $10 \mu \mathrm{g} / \mathrm{mL} \mathrm{IL-4,} \mathrm{IFN-} \gamma$, and TGF- $\beta$ neutralizing antibodies (Bio X Cell) in 10\% FBS-RPMI for 72 hours.

Blocking assay. Hamster anti-mouse IL-1 $\beta$ antibody (Bio X Cell) was injected intravenously $(1 \mu \mathrm{g} / \mathrm{g}$ or $10 \mu \mathrm{g} / \mathrm{g}$ weight $)$ at the indicated timepoints.

Human MDMs and MDDCs. To obtain monocytes, PBMCs were cultured in tissue culture plates at a seeding density of $1 \times 10^{6}$ cells/ $\mathrm{mL}$ in RP-10 media (RPMI-1640 medium [Invitrogen] with 10\% FBS [Atlanta Biologicals] and $2 \mathrm{mM}$ L-glutamine) supplemented with 100 ng/mL GM-CSF (PeproTech) plus 50 ng/mL IL-4 (PeproTech) (MDDCs) at $37^{\circ} \mathrm{C}$ with $5 \% \mathrm{CO}_{2}$. After 4 days, the plates were washed with Hanks' balanced salt solution devoid of divalent cations (Invitrogen) to remove nonadherent cells. Adherent cells were then trypsinized, pelleted, and cultured for 10 days. To assess their function, MDDCs were treated with poly I-C (MilliporeSigma), infected with MERS-CoV-2 (MOI = 2) or mock infected (DMEM) and the expression of cytokine RNAs was determined at 24 hours after infection.

CRISPR-Cas9 editing. Human MDDCs were treated with group IID sPLA2 HDR plasmid (sc-411908-HDR) or control plasmid as per manufacturer's instructions (Santa Cruz Biotechnology, Inc). Briefly, cells were plated at $2 \times 10^{6}$ in $3 \mathrm{~mL}$ antibiotic-free RP-10 per well in a 6-well plate, 24 hours before transfection. For each transfection, 1.5 $\mu \mathrm{g}$ plasmid DNA was diluted with transfection medium to bring the final volume to $150 \mu \mathrm{L}$ and mixed with transfection reagent prior to dropwise addition to cells. Cells were incubated for 72 to 96 hours. The efficacy of editing was determined by PCR.

Cytokine mRNA analysis. Total RNA was extracted from MDDCs using Trizol (Invitrogen) according to the manufacturer's protocol and used for RT-PCR analysis as previously described (46). Following DNase treatment, 200 ng total RNA was used as a template for first strand cDNA. The resulting cDNA was subjected to amplification of selected genes by real-time qPCR using Power SYBR Green PCR Master Mix (Applied Biosystems). Cycle threshold values were normalized to those of the housekeeping gene hypoxanthine phosphoribosyltransferase (HPRT) by the following equation: $\Delta \mathrm{Ct}=\mathrm{Ct}_{\text {(gene of interest) }}-\mathrm{Ct}_{(\mathrm{HPRT})}$. All results are shown as a ratio to HPRT calculated as $2^{-(\mathrm{DCt})}$. The following primers were used for cytokine mRNA detection: IFNA: 5'-AGA AGG CTC CAG CCA TCT CTG T-3', 5'-TGC TGG TAG AGT TCG GTG CAG A-3'; IFNB: 5'-CTT GGA TTC CTA CAA AGA AGC AGC-3', $5^{\prime}$-TCC TCC TTC TGG AAC TGC TGC A-3'; TNFA: 5' - CTC TTC TGC CTG CTG CAC TTT G - $3^{\prime}, 5^{\prime}$ - ATG GGC TAC AGG CTT GTC ACT C -3'; IL1B: $5^{\prime}$ - CCA CAG ACC TTC CAG GAG AAT G -3', 5' - GTG CAG TTC AGT GAT CGT ACA GG -3'; IL6: 5'AGA CAG CCA CTC ACC TCT TCA G - $3^{\prime}, 5^{\prime}$ - TTC TGC CAG TGC CTC TTT GCT G -3'; IL10: 5' - TCT CCG AGA TGC CTT CAG CAG A -3', 5' TCA GAC AAG GCT TGG CAA CCC A - $3^{\prime}$.

Statistics. A Student's $t$ test or 1-way ANOVA with Turkey's post hoc correction was used to analyze differences in mean values between groups. Multiple regression analysis was used to test the repeated measurements between different groups adjusted for time after infection or immunization. Differences in mortality were analyzed using Kaplan-Meier log-rank survival tests. All results are expressed as mean \pm SEM. $P$ values of less than 0.05 were considered statistically significant. ${ }^{*} P<0.05 ;{ }^{* *} P<0.01 ;{ }^{* *} P<0.001$. 
Study approval. All animal studies were approved by the University of Iowa Animal Care and Use Committee and met the requirements of the Guide for the Care and Use of Laboratory Animals. Human peripheral blood samples were obtained from anonymous donors at the DeGowin Blood Center at the University of Iowa. Consent forms were approved by the University of Iowa's Institutional Review Board.

\section{Author contributions}

JZ and SP conceived the work and designed the experiments. JZ and LYRW acquired the data. JZ, DM, and SP analyzed the data. MM and MG provided reagents. JZ, DM, and SP wrote the manuscript.

\section{Acknowledgments}

We thank Kevin Legge, Noah Butler, Alan Sariol, Rudragouda Channappanavar and Rahul Vijay for critical review of the manuscript and members of the Perlman laboratory for helpful discussions. Supported in part by grants from the NIH (RO1 AI129269; PO1 AI060699) (to SP).

Address correspondence to: Stanley Perlman, Department of Microbiology and Immunology, BSB 3-712, University of Iowa, 51 Newton Road, Iowa City, Iowa 52242, USA. Phone: 319.335.8549; Email: Stanley-Perlman@uiowa.edu.
1. World Health Organization. WHO Coronavirus Dashboard, https://covid19.who.int. Accessed May 11, 2021.

2. Hui DS, et al. Middle East respiratory syndrome coronavirus: risk factors and determinants of primary, household, and nosocomial transmission. Lancet Infect Dis. 2018;18(8):e217-e227.

3. Peiris JS, et al. Severe acute respiratory syndrome. Nat Med. 2004;10(12 Suppl):S88-S97.

4. Wu JT, et al. Estimating clinical severity of COVID-19 from the transmission dynamics in Wuhan, China. Nat Med. 2020;26(4):506-510.

5. Memish ZA, et al. Middle East respiratory syndrome. Lancet. 2020;395(10229):1063-1077.

6. De la Fuente M, Miquel J. An update of the oxidation-inflammation theory of aging: the involvement of the immune system in oxi-inflamm-aging. Curr Pharm Des. 2009;15(26):3003-3026.

7. Tuder RM, Petrache I. Pathogenesis of chronic obstructive pulmonary disease. JClin Invest. 2012;122(8):2749-2755.

8. Cannizzo ES, et al. Age-related oxidative stress compromises endosomal proteostasis. Cell Rep. 2012;2(1):136-149.

9. Caruso C, et al. Mechanisms of immunosenescence. Immun Ageing. 2009;6:10.

10. Herrero-Fernandez I, et al. Effect of homeostatic $\mathrm{T}$-cell proliferation in the vaccine responsiveness against influenza in elderly people. Immun Ageing. 2019;16:14.

11. Haq K, McElhaney JE. Ageing and respiratory infections: the airway of ageing. Immunol Lett. 2014;162(1 pt b):323-328.

12. Miki Y, et al. Lymphoid tissue phospholipase A2 group IID resolves contact hypersensitivity by driving antiinflammatory lipid mediators. J Exp Med. 2013;210(6):1217-1234.

13. Vijay R, et al. Critical role of phospholipase A2 group IID in age-related susceptibility to severe acute respiratory syndrome-CoV infection. J Exp Med. 2015;212(11):1851-1868.

14. Miki Y, et al. Dual roles of group IID phospholipase A2 in inflammation and cancer. J Biol Chem. 2016;291(30):15588-15601.

15 . Lee N, et al. Effects of early corticosteroid treatment on plasma SARS-associated coronavirus RNA concentrations in adult patients. J Clin Virol. 2004;31(4):304-309.

16. Li K, et al. Mouse-adapted MERS coronavirus causes lethal lung disease in human DPP4 knockin mice. Proc Natl Acad Sci U S A. 2017;114(15):E3119-E3128.

17. Sun J, et al. Generation of a broadly useful model for COVID-19 pathogenesis, vaccination, and treatment. Cell. 2020;182(3):734-743.

18. Hassan AO, et al. A SARS-CoV-2 infection model in mice demonstrates protection by neutralizing antibodies. Cell. 2020;182(3):744-753.

19. Scobey T, et al. Reverse genetics with a fulllength infectious cDNA of the Middle East respiratory syndrome coronavirus. Proc Natl Acad Sci U S A. 2013;110(40):16157-16162.

20. Zhao J, et al. Airway memory CD4(+) T cells mediate protective immunity against emerging respiratory coronaviruses. Immunity. 2016;44(6):1379-1391.

21. Pushko P, et al. Replicon-helper systems from attenuated Venezuelan equine encephalitis virus: expression of heterologous genes in vitro and immunization against heterologous pathogens in vivo. Virology. 1997;239(2):389-401.

22. Burton DR. Antibodies, viruses and vaccines. Nat Rev Immunol. 2002;2(9):706-713.

23. Crotty S. T follicular helper cell biology: a decade of discovery and diseases. Immunity. 2019;50(5):1132-1148.

24. Ueno $\mathrm{H}$, et al. Pathophysiology of $\mathrm{T}$ follicular helper cells in humans and mice. Nat Immunol. 2015;16(2):142-152.

25. Bauquet AT, et al. The costimulatory molecule ICOS regulates the expression of c-Maf and IL-21 in the development of follicular T helper cells and TH-17 cells. Nat Immunol. 2009;10(2):167-175.

26. Lu KT, et al. Functional and epigenetic studies reveal multistep differentiation and plasticity of in vitro-generated and in vivo-derived follicular T helper cells. Immunity. 2011;35(4):622-632.

27. Nurieva RI, et al. Generation of T follicular helper cells is mediated by interleukin-21 but independent of T helper 1, 2, or 17 cell lineages. Immunity. 2008;29(1):138-149.

28. Botta D, et al. Dynamic regulation of T follicular regulatory cell responses by interleukin 2 during influenza infection. Nat Immunol. 2017;18(11):1249-1260

29. Canete PF, et al. Regulatory roles of IL-10-producing human follicular T cells. J Exp Med. 2019;216(8):1843-1856

30. Hou S, et al. FoxP3 and Ezh2 regulate Tfr cell suppressive function and transcriptional program. JExp Med. 2019;216(3):605-620.

31. Liu Z, et al. Immune homeostasis enforced by co-localized effector and regulatory T cells. Nature. 2015;528(7581):225-230.

32. Huang Q, et al. Molecular basis of the differentiation and function of virus specific follicular helper CD4 ${ }^{+}$T cells. Front Immunol. 2019;10:249.

33. Dolence JJ, et al. Airway exposure initiates peanut allergy by involving the IL-1 pathway and T follicular helper cells in mice. J Allergy Clin Immunol. 2018;142(4):1144-1158.

34. Ritvo PG, et al. $\mathrm{T}_{\mathrm{fr}}$ cells lack IL-2R $\alpha$ but express decoy IL-1R2 and IL-1Ra and suppress the IL-1-dependent activation of $\mathrm{T}_{\mathrm{fh}}$ cells. Sci Immunol. 2017;2(15):eaan0368.

35. Barbet $\mathrm{G}$, et al. Sensing microbial viability through bacterial RNA augments $t$ follicular helper cell and antibody responses. Immunity. 2018;48(3):584-598.

36. Ritvo PG, Klatzmann D. Interleukin-1 in the response of follicular helper and follicular regulatory T cells. Front Immunol. 2019;10:250.

37. McCurdy S, et al. Macrophage-specific expression of IL-37 in hyperlipidemic mice attenuates atherosclerosis. JImmunol. 2017;199(10):3604-3613.

38. Yang J, et al. IL-6 impairs vaccine responses in neonatal mice. Front Immunol. 2018;9:3049.

39. Davies FJ, et al. Streptococcal superantigen-induced expansion of human tonsil T cells leads to altered $\mathrm{T}$ follicular helper cell phenotype, B cell death and reduced immunoglobulin release. Clin Exp Immunol. 2019;197(1):83-94.

40. Jain A, et al. T cells instruct myeloid cells to produce inflammasome-independent IL-1 $\beta$ and cause autoimmunity. Nat Immunol. 2020;21(1):69-74

41. Kaneko N, et al. The loss of Bcl-6 expressing T follicular helper cells and the absence of germinal centers in COVID-19. Cell. 2020;183(1):143-157.

42. Hammad $\mathrm{H}$, et al. Activation of the $\mathrm{D}$ prostanoid 1 receptor suppresses asthma by modulation of lung dendritic cell function and induction of regulatory T cells. J Exp Med. 2007;204(2):357-367.

43. Fehr AR. Bacterial artificial chromosome-based lambda red recombination with the I-SceI homing endonuclease for genetic alteration of MERSCoV. Methods Mol Biol. 2020;2099:53-68.

44. Zheng J, Perlman S. Immune responses in influenza A virus and human coronavirus infections: an ongoing battle between the virus and host. Curr Opin Virol. 2018;28:43-52.

45. Meyerholz DK, et al. Approaches to evaluate lung inflammation in translational research. Vet Pathol. 2018;55(1):42-52.

46. Pewe L, et al. A severe acute respiratory syndrome-associated coronavirus-specific protein enhances virulence of an attenuated murine coronavirus. J Virol. 2005;79(17):11335-11342.

47. Ainsua-Enrich E, et al. IRF4-dependent dendritic cells regulate $\mathrm{CD} 8^{+} \mathrm{T}$-cell differentiation and memory responses in influenza infection. $\mathrm{Muco}$ sal Immunol. 2019;12(4):1025-1037. 